\title{
Three-body treatment of the penetration through the Coulomb field of a two-fragment nucleus
}

\author{
V.F. Kharchenko ${ }^{1}$ and A.V. Kharchenko \\ Bogolyubov Institute for Theoretical Physics, National Academy of Sciences of Ukraine, \\ UA - 03143, Kyiv, Ukraine
}

\begin{abstract}
On the basis of the Faddeev integral equations method and the Watson- Feshbach concept of the effective (optical) interaction potential, the first fully consistent threebody approach to the description of the penetration of a charged particle through the Coulomb field of a two-particle bound complex (composed of one charged and one neutral particles) has been developed. A general formalism has been elaborated and on its basis, to a first approximation in the Sommerfeld parameter, the influence of the nuclear structure on the probability of the penetration of a charged particle (the muon, the pion, the kaon and the proton) through the Gamow barrier of a two-fragment nucleus (the deuteron and the two lightest lambda hypernuclei, ${ }_{\Lambda}^{3} \mathrm{H}$ and ${ }_{\Lambda}^{5} \mathrm{He}$ ) has been calculated and studied.
\end{abstract}

PACS: 03.65.Nk; 21.45.+v; 21.80.+a

Keywords: Three-body formalism; Coulomb interaction; Gamow barrier of two-fragment nucleus; Penetration factor; Deuteron; Lambda hypernuclei ${ }_{\Lambda}^{3} \mathrm{H}$ and ${ }_{\Lambda}^{5} \mathrm{He}$

\footnotetext{
${ }^{1}$ E-mail: vkharchenko@bitp.kiev.ua
} 


\section{Introduction}

The real nuclear systems, as a rule, contain charged particles. The interplay of the finite-range nuclear and long-range Coulomb forces is one of the fundamental peculiarities of the nuclear systems that is responsible for occuring specific phenomena.

The Gamow theory of the radioactive decay of heavy nuclei $[1,2]$ was among first advantageous applications of the quantum mechanics to the description of atomic nuclei. According to the theory, the probability of the alpha-decay of a nucleus is essentially determined by the probability of the penetration of the $\alpha$-particle from the inside of the nucleus where the nuclear force dominates to the outside through the Coulomb barrier of the nucleus. Presence of the Coulomb interaction between particles can also effect essentially on characteristics of nuclear processes involving charged particles, most pronouncedly at low energies.

Of particular interest is the investigation of the effects of the Coulomb interaction for the simplest nuclear systems at the microscopic level — on the basis of the rigorous few-body equations. The application of the few-body approach to the problem of scattering a charged particle by the Coulomb field of a composite bound system containing charged particles makes it possible to reveal new regularities for processes initiated by the collision. In particular, this approach enables one to investigate the influence of the structure of the scattering complex on the magnitude of the penetration factor for the Coulomb field of the complex.

It is known [3] that the Faddeev integral equations for three particles [4] with the Coulomb interaction between the particles are non-Fredholm at energies above the threshold of the elastic scattering of a particle on a two-particle bound system and therefore they must be rebuilt. For the problem of the scattering of two charged particles, a convenient method of solving the non-Fredholm LippmannSchwinger integral equation with the Coulomb potential that is based on using the limiting transition from the equation with the screened Coulomb potential has been elaborated by Gorshkov [5]. Because of the non-Fredholm character of the kernel of the two-particle integral equation with the "pure" Coulomb potential (in threedimensional formulation), the solution of the scattering problem for two charged particles is known not to be the immediate limiting case of the solution of the problem with the screened Coulomb potential when the screening radius $R_{s}$ is going to infinity. The Gorshkov recipe of the transition consists in extraction explicitly from the wave function of the system with the screened Coulomb potential of the known exponential Coulomb factor, singular at $R_{s} \rightarrow \infty$. The generalization of the 
Gorshkov's recipe for the problem of the scattering of a charged particle on a bound system consisting of two charged particles has been proposed by Vesselova [6].

The appropriate regularized integral equations of the proton-deuteron scattering problem were obtained in the paper [7]. In the frame of the quasi-particle AGS formalism [8] the Vesselova method has been also used in the papers $[9,10]$. A further numerical calculations have shown the benefit from a correct accounting of the Coulomb effects (especially in the "inner" range - in the range of the deuteron system) using the integral equations method in the momentum space $[9,11-13]$ as well as the integro-differential equations method in the configuration space [14].

As a result of the analytical study of the effective interaction between a charged particle and two-particle complex on the basis of the Faddeev integral equations, it has been established that the full concellation of the long-range terms proportional to $\left(e_{1} e_{2}\right)^{2}\left|\mathbf{p}-\mathbf{p}^{\prime}\right|^{-1},\left(e_{1} e_{2}\right)^{3} \ln \left(\left|\mathbf{p}-\mathbf{p}^{\prime}\right| \rho_{0}\right)$ and $\left(e_{1} e_{2}\right)^{4}\left|\mathbf{p}-\mathbf{p}^{\prime}\right|$ occur when the transfer momentum tends to zero $\left(\left|\mathbf{p}-\mathbf{p}^{\prime}\right| \rightarrow 0\right)$ and the behaviour of the polarization potential at asymptotically large [15-18] and intermediate [19-20] distances has been determined in an explicit form.

The advance in elaboration of the description of the three-particle system containing charged particles makes it possible to formulate and investigate the problem on the penetration of a charged particle through the Coulomb field of a two-particle bound system on the mathematically rigorous level.

In this paper we develop the first fully consistent three-body approach to the description of the penetration of a charged particle through the Coulomb field of a two-fragment bound nucleus. In Section 2 we begin with formulation of this problem as a three-body problem on the Coulomb scattering of a charged particle by a two-body bound system composed of one charged and one neutral particles. Using the Watson-Feshbach concept of the effective potential we reduce in a rigorous way the three-body problem to a definite two-body scattering problem. In Section 3 we elaborate a three-body formalism for the description of the penetration of a charged particle through the Coulomb field of the two-body nuclear complex. We derive a general formula for the relative difference between the penetration factor for the Gamow barrier of a two-fragment nucleus and the penetration factor for the Coulomb field of the corresponding point charge and study its behaviour on simplifying assumptions. In Section 4 we calculate the effect of influence of the structure of two-fragment nuclei (the deuteron and the lambda-hypernuclei ${ }_{\Lambda}^{3} \mathrm{H}$ and ${ }_{\Lambda}^{5} \mathrm{He}$ ) on the probabilities of penetration of incident charged particles (the meson, the pion, the kaon and the proton) through the Gamow barriers of the nuclei. We summarize our results in Section 5. 


\section{Basic equations for the scattering of a charged particle by the Coulomb field of a two-particle complex}

Let us consider the three-particle problem on the scattering of a charged particle 1 by a bound complex that consists of two particles - a charged particle 2 and a neutral particle 3 . (The particle $i$ is characterized by the mass $m_{i}$ and the charge $\left.e_{i}.\right)$ In this paper we restrict our consideration to a three-particle system with two pair interaction potentials - a short-range (nuclear) potential describing the interaction between the particles 2 and $3, v_{23}^{N}$, that provides formation a bound twoparticle complex, and the long-range Coulomb potential of the interaction between the particles 1 and $2, v_{12}^{C}$. We regard that the particles 1 and 3 do not interact at all, $v_{31}=0$. The operator of the potential interaction energy for the considered system of three particles has the form

$$
V=v_{12}^{C}+v_{23}^{N}
$$

Note, that the description of the three particle system with the potential (1) is the simplest three-particle Coulomb problem. This problem can have an independent physical interest or arise up as an auxiliary problem when finding the integral kernels of the modified Faddeev equations for the system containing charged particles $[21]$.

\subsection{Three-particle equations with the screened Coulomb interaction}

To derive the integral equations for the scattering of a charged particle on a two-particle complex consisting of a charged and neutral particles, we start from the interaction operator for the three-particle system $\bar{V}$ that contains the screened Coulomb pair potential $\bar{v}_{12}^{C}$,

$$
\bar{V}=\bar{v}_{12}^{C}+v_{23}^{N}, \quad \bar{v}_{12}^{C}=v_{12}^{C} \cdot s_{12},
$$

where $v_{12}^{C}$ is the ordinary "pure" Coulomb potential, $v_{12}^{C}\left(r_{12}\right)=e_{1} e_{2} / r_{12}$, and $s_{12}$ is a screening function that can be chosen in the exponential form: $s_{12}\left(r_{12}, R_{s}\right)=$ $\exp \left(-r_{12} / R_{s}\right), R_{s}$ is a parameter that describes the screening distance. Here and further we mark all quantities, which correspond to the screened Coulomb potential, by bar.

Splitting (correspondingly to the form of the potential operator (2)) the total wave function of the three-particle system $\bar{\Psi}$ into two Faddeev's components,

$$
\bar{\Psi}=\bar{\Psi}^{(23)}+\bar{\Psi}^{(12)}
$$


for the components we obtain the system of the coupled integral equations:

$$
\begin{aligned}
& \bar{\Psi}^{(23)}=\Phi_{23,1}+G_{0}(E) T_{23}(E) \bar{\Psi}^{(12)}, \\
& \bar{\Psi}^{(12)}=\quad G_{0}(E) \bar{T}_{12}^{C}(E) \bar{\Psi}^{(23)} .
\end{aligned}
$$

Here, the operator $G_{0}(E)=\left(E-h_{0}^{23}-h_{0}^{1}+i 0\right)^{-1}$ is the free three-particle propagator, $\left(h_{0}^{23}\right.$ and $h_{0}^{1}$ are the operators of the kinetic energy of the relative motion of the particles 2 and 3 and that of the particle 1 and the centre of mass of the particles 2 and 3$), E$ is the total energy of the relative motion in the system of three particles, $\bar{T}_{12}^{C}(E)$ and $T_{23}(E)$ are the transition operators that correspond to the pair interactions $\bar{v}_{12}^{C}$ and $v_{23}$, respectively, and satisfy to the Lippmann-Schwinger equations with the free three-particle propagator $G_{0}(E)$,

$$
\begin{aligned}
& \bar{T}_{12}^{C}(E)=\bar{v}_{12}^{C}+\bar{v}_{12}^{C} G_{0}(E) \bar{T}_{12}^{C}(E), \\
& T_{23}(E)=v_{23}+v_{23} G_{0}(E) T_{23}(E) .
\end{aligned}
$$

The free term in Eq. (4) is the product of the wave function of the bound state of the two-particle complex $\psi_{0}$ and the plane wave of the relative motion of the incident particle 1 and the centre of mass of two particles of the complex with the momentum $\mathbf{p}_{0}, \quad \varphi_{\mathbf{p}_{0}}=\left|\mathbf{p}_{0}\right\rangle$,

$$
\Phi_{23,1}=\psi_{0} \cdot \varphi_{\mathbf{p}_{0}}
$$

As variables that describe the three-particle system we shall use the relative Jacoby momenta:

$$
\mathbf{k}_{i j}=\left(m_{j} \mathbf{k}_{i}-m_{i} \mathbf{k}_{j}\right) / m_{i j}, \quad \mathbf{p}_{k}=\left[m_{k}\left(\mathbf{k}_{i}+\mathbf{k}_{j}\right)-m_{i j} \mathbf{k}_{k}\right] / M,
$$

where $\mathbf{k}_{i}$ is the momentum of the particle $i, m_{i j}=m_{i}+m_{j}, \quad M=m_{1}+m_{2}+$ $m_{3}, \quad i j k=123,231,312$. In the configuration space the relative coordinates

$$
\mathbf{r}_{i j}=\mathbf{r}_{i}-\mathbf{r}_{j}, \quad \boldsymbol{\rho}_{k}=\left(m_{i} \mathbf{r}_{i}+m_{j} \mathbf{r}_{j}\right) / m_{i j}-\mathbf{r}_{k},
$$

where $\mathbf{r}_{i}$ is the radius-vector of the particle $i$, correspond to the relative momenta (7). Below we shall use the short-cut definitions of the momentum variables $\mathbf{k} \equiv$ $\mathbf{k}_{23}, \quad \mathbf{p} \equiv \mathbf{p}_{1} \quad$ and the coordinates $\mathbf{r} \equiv \mathbf{r}_{23}, \quad \boldsymbol{\rho} \equiv \boldsymbol{\rho}_{1}$.

For the problem on the scattering of a particle on a two-particle complex the total energy of the relative motion in the three-particle system is equal to

$$
E=\epsilon-b
$$

where $\epsilon=p_{0}^{2} / 2 \mu_{1}$ is the energy of the relative motion of the particle 1 and the centre of mass of the complex of the particles 2 and $3, b=\kappa^{2} / 2 \mu_{23}$ is the binding energy of 
the two-particle complex, $\mu_{i j}=m_{i} m_{j} / m_{i j} \quad$ and $\mu_{k}=m_{k} m_{i j} / M$ are the reduced masses of two particles $i$ and $j$ and of the particle $k$ and the centre of mass of the pair of particles, $i$ and $j$, respectively.

For simplicity sake the interaction between particles 2 and 3 that form a bound complex will be described by the separable potential:

$$
v_{23}^{N}=-\lambda|u\rangle\langle u|
$$

To the potential (9) there corresponds the two-particle transition operator

$$
t_{23}^{N}(\varepsilon)=|u\rangle \tau(\varepsilon)\langle u|
$$

where

$$
\tau(\varepsilon)=\frac{S(\varepsilon)}{\varepsilon+b}, \quad S(\varepsilon)=\left\langle u\left|g_{0}^{23}(-b) g_{0}^{23}(\varepsilon)\right| u\right\rangle^{-1}, g_{0}^{23}(\varepsilon)=\left(\varepsilon-h_{0}^{23}+i 0\right)^{-1},
$$

$\varepsilon$ is the energy of the relative motion of the particles 2 and 3 . The wave function of the bound $S$-state of the two particles has the form

$$
\left|\psi_{0}\right\rangle=-g_{0}^{23}(-b)|u\rangle, \quad \psi_{0}(\mathbf{k})=u(k)\left(\frac{k^{2}}{2 \mu_{23}}+b\right)^{-1}, \quad\left\langle\psi_{0} \mid \psi_{0}\right\rangle=1,
$$

in this case the occurence of the bound state of two particles implies the fulfilment of the condition

$$
\frac{1}{\lambda}+\left\langle u\left|g_{0}^{23}(-b)\right| u\right\rangle=0
$$

In the case of the potential (9) the transition operator $T_{23}(E)$ in the equation of the set (4) that is determined by the Lippmann-Schwinger equation (5) with the three-particle free propagator $G_{0}(E)$, takes the form

$$
T_{23}(E)=|u\rangle \tau\left(E-h_{0}^{1}\right)\langle u|, \quad \tau\left(E-h_{0}^{1}\right)=S\left(E-h_{0}^{1}\right) g_{0}^{1}(E) .
$$

Note, that the expression (14) for $T_{23}(E)$ contains a diagonal operator in the functional space of a relative momentum of the particle 1 and the centre of mass of the particles 2 and 3 .

By applying the interaction model (9) the formal solution of the set of equations (4) can be written as

$$
\begin{aligned}
\bar{\Psi}^{(23)} & =-G_{0}(E)|u\rangle\left[1+\tau\left(E-h_{0}^{1}\right) \bar{X}(\epsilon)\right] \varphi_{\mathbf{p}_{0}}, \\
\bar{\Psi}^{(12)} & =-G_{0}(E) \bar{T}_{12}^{C}(E) G_{0}(E)|u\rangle\left[1+\tau\left(E-h_{0}^{1}\right) \bar{X}(\epsilon)\right] \varphi_{\mathbf{p}_{0}},
\end{aligned}
$$

where the operator $\bar{X}(\epsilon)$ satisfies the integral equation

$$
\begin{aligned}
\bar{X}(\epsilon)= & \bar{U}(\epsilon)+\bar{U}(\epsilon) \tau\left(\epsilon-b-h_{0}^{1}\right) \bar{X}(\epsilon), \\
& \bar{U}(\epsilon)=\left\langle u\left|G_{0}(\epsilon-b) \bar{T}_{12}^{C}(\epsilon-b) G_{0}(\epsilon-b)\right| u\right\rangle .
\end{aligned}
$$


Taking into account Eq. (15) we write the complete wave function (3) in the form

$$
\bar{\Psi}=-\left[1+G_{0}(E) \bar{T}_{12}^{C}(E)\right] G_{0}(E)|u\rangle\left[1+\tau\left(E-h_{0}^{1}\right) \bar{X}(\epsilon)\right] \varphi_{\mathbf{p}_{0}} .
$$

The operators $\bar{X}(\epsilon), \bar{U}(\epsilon)$ and $\tau\left(\epsilon-b-h_{0}^{1}\right)$ in Eqs. (15) and (16) act in the space of the functions of the variables that describe only the relative motion of the particle 1 and the centre of mass of the particles 2 and 3 - the momentum vector $\mathbf{p}_{1}$ or the radius-vector $\boldsymbol{\rho}_{1}$. With the formulae (15) - (16), the three-body problem under study is reduced to a definite two-body problem on the motion of the particle 1 relative to the centre of mass of the particles 2 and 3 . The latter is formulated in the form of the integral equation (16). The attained simplification of the three-body problem, feasible because of using a specific model of the interaction between the particles of the bound complex (9), is rigorous and can be generalized to other models of the finite-range interaction.

\subsection{Equation for the scattering wave function of a reduced two-body problem}

Instead of the operator $\bar{X}(\epsilon)$ (matrix elements of which are contained in the components of the wave function (15) with the initial momentum on the energy shell $\epsilon$ ) it is convenient to use the relative wave function

$$
\bar{\chi}_{\mathbf{p}_{0}}=\left[1+g_{0}^{1}(\epsilon) \bar{X}(\epsilon)\right] \varphi_{\mathbf{p}_{0}} .
$$

The integral equation for the function $\bar{\chi}_{\mathbf{p}_{0}}$ that follows from Eqs. (16) and (18) has the form

$$
\bar{\chi}_{\mathbf{p}_{0}}=\varphi_{\mathbf{p}_{0}}+g_{0}^{1}(\epsilon) \bar{U}(\epsilon) S\left(\epsilon-b-h_{0}^{1}\right) \bar{\chi}_{\mathbf{p}_{0}} .
$$

With the use of the function $\bar{\chi}_{\mathbf{p}_{0}}$ and the relation

$$
\left[1+\tau\left(\epsilon-b-h_{0}^{1}\right) \bar{X}(\epsilon)\right] \varphi_{\mathbf{p}_{0}}=S\left(\epsilon-b-h_{0}^{1}\right) \bar{\chi}_{\mathbf{p}_{0}}
$$

the expressions for the Faddeev's components (15) and the complete wave functions (17) take the form

$$
\begin{aligned}
& \bar{\Psi}^{(23)}=-G_{0}(E)|u\rangle S\left(\epsilon-b-h_{0}^{1}\right) \bar{\chi}_{\mathbf{p}_{0}}, \\
& \bar{\Psi}^{(12)}=-G_{0}(E) \bar{T}_{12}^{C}(E) G_{0}(E)|u\rangle S\left(\epsilon-b-h_{0}^{1}\right) \bar{\chi}_{\mathbf{p}_{0}}
\end{aligned}
$$

and

$$
\bar{\Psi}=-\left[1+G_{0}(E) \bar{T}_{12}^{C}(E)\right] G_{0}(E)|u\rangle S\left(\epsilon-b-h_{0}^{1}\right) \bar{\chi}_{\mathbf{p}_{0}} .
$$

The scattering wave function for the effective two-body problem on the motion of the charged particle 1 relative to the centre of mass of the particles of the complex, 
$\bar{\psi}_{\mathbf{p}_{0}}^{e f f}(1)$, we determine following the method of introducing the effective interaction between a particle and a complex by Francis-Watson [22] and Feshbach [23] (see also Refs. [24] and [25]) as a coefficient function before the wave function of the bound state of the two-body complex in the expansion of the complete three-particle wave function in the set of the two-particle wave functions of the relative motion of the interacting particles 2 and 3 ,

$$
\bar{\psi}_{\mathbf{p}_{0}}^{e f f}(1) \equiv\left\langle\psi_{0}(23) \mid \bar{\Psi}_{\mathbf{p}_{0}}(23,1)\right\rangle
$$

In the momentum space of variables the formula (23) has the form

$$
\bar{\psi}_{\mathbf{p}_{0}}^{\text {eff }}(\mathbf{p})=\int \frac{d \mathbf{k}}{(2 \pi)^{3}} \psi_{0}^{*}(\mathbf{k}) \bar{\Psi}_{\mathbf{p}_{0}}(\mathbf{k}, \mathbf{p}) .
$$

Taking into account the explicit form of the total wave function (22) in the expression (23), we obtain the relation between the effective wave function $\bar{\psi}_{\mathbf{p}_{0}}^{e f f}$ and the above-introduced function $\bar{\chi}_{\mathbf{p}_{0}}$,

$$
\bar{\psi}_{\mathbf{p}_{0}}^{e f f}=\left[1+\bar{Z}(\epsilon) S\left(\epsilon-b-h_{0}^{1}\right)\right] \bar{\chi}_{\mathbf{p}_{0}}
$$

where

$$
\bar{Z}(\epsilon)=-\left\langle\psi_{0}\left|G_{0}(\epsilon-b) \bar{T}_{12}^{C}(\epsilon-b) G_{0}(\epsilon-b)\right| u\right\rangle .
$$

The expression (26) can be written as

$$
\bar{Z}(\epsilon)=g_{0}(\epsilon)[\bar{W}(\epsilon)-\bar{U}(\epsilon)]
$$

where

$$
\bar{W}(\epsilon)=\left\langle u\left|g_{0}^{23}(-b) \bar{T}_{12}^{C}(\epsilon-b) G_{0}(\epsilon-b)\right| u\right\rangle,
$$

and the operator $\bar{U}(\epsilon)$ is determined by the expression (16). The integration in the expressions for $\bar{Z}(\epsilon)$ and $\bar{W}(\epsilon)$, as in the expression (16) for $\bar{U}(\epsilon)$, is taken only in the space of variables of the relative motion of the particles inside the complex.

Introducing the effective transition operator $\bar{X}_{\text {eff }}(\epsilon)$ that corresponds to the effective wave function

$$
\bar{\psi}_{\mathbf{p}_{0}}^{e f f}=\left[1+g_{0}^{1}(\epsilon) \bar{X}_{e f f}(\epsilon)\right] \varphi_{\mathbf{p}_{0}}
$$

and taking into account the relations (25), (27) (18), we obtain the formula expressing the transition operator $\bar{X}_{e f f}(\epsilon)$ through the operators $\left.\bar{X}_{(} \epsilon\right)$ and $\bar{W}(\epsilon)$,

$$
\bar{X}_{e f f}(\epsilon)=\bar{W}(\epsilon)+\bar{W}(\epsilon) \tau\left(\epsilon-b-h_{0}^{1}\right) \bar{X}(\epsilon) .
$$




\subsection{The passage to the unscreened Coulomb interaction}

The removal of the screening of the Coulomb interaction between charged particles (the passage to the unlimitedly great screening distance, $R_{s} \rightarrow \infty$ ) in Eq. (19), that determines the scattering wave function of the reduced two-particle problem and the effective wave function (24), we perform by applying the known Gorshkov-Vesselova recipe $[5,6]$. Notice that the quantities describing the system with the unscreened Coulomb interaction we denote by the same letters but without overscribed bar.

The kernels of the operators $\bar{U}, \bar{W}$ and $\bar{Z}$ in the integral equations (19) and (30) and in the expression for the effective wave function (25), which are determined by the formulae (16),(26) and (28), has the form

$$
\begin{aligned}
\bar{U}\left(\mathbf{p}, \mathbf{p}^{\prime} ; \epsilon\right) & =\int \frac{d \mathbf{k}}{(2 \pi)^{3}} \frac{u(\mathbf{k})\left\langle\mathbf{k}_{12}\left|\bar{t}_{12}^{C}\left(\epsilon-b-\frac{p_{3}^{2}}{2 \mu_{3}}\right)\right| \mathbf{k}_{12}^{\prime}\right\rangle u(\mathbf{k}-2 \mathbf{Q})}{\left[\frac{k^{2}}{2 \mu_{23}}+b-\epsilon_{p}\right]\left[\frac{(\mathbf{k}-2 \mathbf{Q})^{2}}{2 \mu_{23}}+b-\epsilon_{p^{\prime}}\right]}, \\
\bar{W}\left(\mathbf{p}, \mathbf{p}^{\prime} ; \epsilon\right) & =\int \frac{d \mathbf{k}}{(2 \pi)^{3}} \frac{u(\mathbf{k})\left\langle\mathbf{k}_{12}\left|\bar{t}_{12}^{C}\left(\epsilon-b-\frac{p_{3}^{2}}{2 \mu_{3}}\right)\right| \mathbf{k}_{12}^{\prime}\right\rangle u(\mathbf{k}-2 \mathbf{Q})}{\left[\frac{k^{2}}{2 \mu_{23}}+b\right]\left[\frac{(\mathbf{k}-2 \mathbf{Q})^{2}}{2 \mu_{23}}+b-\epsilon_{p^{\prime}}\right]} \\
\bar{Z}\left(\mathbf{p}, \mathbf{p}^{\prime} ; \epsilon\right) & =-\int \frac{d \mathbf{k}}{(2 \pi)^{3}} \frac{u(\mathbf{k})\left\langle\mathbf{k}_{12}\left|\bar{t}_{12}^{C}\left(\epsilon-b-\frac{p_{3}^{2}}{2 \mu_{3}}\right)\right| \mathbf{k}_{12}^{\prime}\right\rangle u(\mathbf{k}-2 \mathbf{Q})}{\left[\frac{k^{2}}{2 \mu_{23}}+b\right]\left[\frac{k^{2}}{2 \mu_{23}}+b-\epsilon_{p}\right]\left[\frac{(\mathbf{k}-2 \mathbf{Q})^{2}}{2 \mu_{23}}+b-\epsilon_{p^{\prime}}\right]},
\end{aligned}
$$

where for simplicity sake the following designations are used:

$$
\begin{gathered}
\mathbf{k}_{12}=-\frac{m_{1}}{m_{12}} \mathbf{p}_{3}-\mathbf{p}, \quad \mathbf{k}_{12}^{\prime}=-\frac{m_{1}}{m_{12}} \mathbf{p}_{3}-\mathbf{p}^{\prime}, \quad \mathbf{p}_{3}=\mathbf{k}-\frac{m_{3}}{m_{23}} \mathbf{p} \\
\mathbf{Q}=\frac{m_{3}}{2 m_{23}}\left(\mathbf{p}-\mathbf{p}^{\prime}\right), \quad \epsilon_{p}=\epsilon-\frac{p^{2}}{2 \mu_{1}}+i 0
\end{gathered}
$$

In the limiting case of the unscreened ("pure") Coulomb interaction $\left(R_{s} \rightarrow \infty\right.$ : $\left.\bar{v}_{12}^{C} \rightarrow v_{12}^{C}\right)$ the two-particle Coulomb transition matrix $\bar{t}_{12}^{C} \rightarrow t_{12}^{C}$ ) in the integrable expressions (31) generates long-ranged (Coulomb and polarization) interactions between the charged incident particle and the centre of mass of complex. The most

long-ranged part of the kernels of the operators $\{U, W, Z\}=\lim _{R_{s} \rightarrow \infty}\{\bar{U}, \bar{W}, \bar{Z}\}$ is formed in Eq. (31) from the Born term of the two-particle Coulomb transition matrix. The corresponding matrix element being local depends on $\mathbf{k}_{12}^{\prime}-\mathbf{k}_{12}=\mathbf{p}-\mathbf{p}^{\prime}$, and hence is a function of only variables characterizing the relative motion of the particle 1 and the centre of mass of two other particles, which has the form of the screened Coulomb potential of interaction between the point charges of the particle 
$1\left(e_{1}\right)$ and the centre of mass of the two-body complex $\left(e_{2}\right), \bar{V}^{C}\left(\mathbf{p}-\mathbf{p}^{\prime}\right)$,

$$
\left\langle\mathbf{k}_{12}\left|\bar{t}_{12}^{C}\left(\epsilon-b-\frac{p_{3}^{2}}{2 \mu_{3}}\right)\right| \mathbf{k}_{12}^{\prime}\right\rangle_{\text {Born }}=\bar{v}_{12}^{C}\left(\mathbf{k}_{12}-\mathbf{k}_{12}^{\prime}\right)=\frac{4 \pi e_{1} e_{2}}{\left(\mathbf{p}-\mathbf{p}^{\prime}\right)^{2}+R_{s}^{2}}=\bar{V}^{C}\left(\mathbf{p}-\mathbf{p}^{\prime}\right)
$$

and is taken outside the integral signs in Eqs. (31),

$$
\begin{aligned}
\bar{U}\left(\mathbf{p}, \mathbf{p}^{\prime} ; \epsilon\right) & =\bar{V}^{C}\left(\mathbf{p}-\mathbf{p}^{\prime}\right) I\left(\mathbf{p}, \mathbf{p}^{\prime} ; \epsilon\right)+\cdots \\
\bar{W}\left(\mathbf{p}, \mathbf{p}^{\prime} ; \epsilon\right) & =\bar{V}^{C}\left(\mathbf{p}-\mathbf{p}^{\prime}\right) J\left(\mathbf{p}, \mathbf{p}^{\prime} ; \epsilon\right)+\cdots \\
\bar{Z}\left(\mathbf{p}, \mathbf{p}^{\prime} ; \epsilon\right) & =\bar{V}^{C}\left(\mathbf{p}-\mathbf{p}^{\prime}\right) L\left(\mathbf{p}, \mathbf{p}^{\prime} ; \epsilon\right)+\cdots
\end{aligned}
$$

where

$$
\begin{aligned}
I\left(\mathbf{p}, \mathbf{p}^{\prime} ; \epsilon\right) & =\int \frac{d \mathbf{k}}{(2 \pi)^{3}} \frac{u(\mathbf{k}) u(\mathbf{k}-2 \mathbf{Q})}{\left[\frac{k^{2}}{2 \mu_{23}}+b-\epsilon_{p}\right]\left[\frac{(\mathbf{k}-2 \mathbf{Q})^{2}}{2 \mu_{23}}+b-\epsilon_{p^{\prime}}\right]}, \\
J\left(\mathbf{p}, \mathbf{p}^{\prime} ; \epsilon\right) & =\int \frac{d \mathbf{k}}{(2 \pi)^{3}} \frac{u(\mathbf{k}) u(\mathbf{k}-2 \mathbf{Q})}{\left[\frac{k^{2}}{2 \mu_{23}}+b\right]\left[\frac{(\mathbf{k}-2 \mathbf{Q})^{2}}{2 \mu_{23}}+b-\epsilon_{p^{\prime}}\right]}, \\
L\left(\mathbf{p}, \mathbf{p}^{\prime} ; \epsilon\right) & =-\int \frac{d \mathbf{k}}{(2 \pi)^{3}} \frac{u(\mathbf{k}) u(\mathbf{k}-2 \mathbf{Q})}{\left[\frac{k^{2}}{2 \mu_{23}}+b\right]\left[\frac{k^{2}}{2 \mu_{23}}+b-\epsilon_{p}\right]\left[\frac{(\mathbf{k}-2 \mathbf{Q})^{2}}{2 \mu_{23}}+b-\epsilon_{p^{\prime}}\right]} .
\end{aligned}
$$

Note, that the factors $I, J$ and $L$ in Eqs. (34) do not contain the screening parameter $R_{s}$, they are essentially non-local and fall rapidly at large values of the momenta $p$ and $p^{\prime}$. In accordance with Eq. (27) there exists a relation between the factors (34) having the form

$$
L\left(\mathbf{p}, \mathbf{p}^{\prime} ; \epsilon\right)=\left(\epsilon-\frac{p^{2}}{2 \mu_{1}}\right)^{-1}\left[J\left(\mathbf{p}, \mathbf{p}^{\prime} ; \epsilon\right)-I\left(\mathbf{p}, \mathbf{p}^{\prime} ; \epsilon\right)\right] .
$$

The diagonal elements $I, J$ and $L$ with the momenta on the energy shell are equal to

$$
\begin{aligned}
I\left(\mathbf{p}_{0}, \mathbf{p}_{0} ; \epsilon\right) & =J\left(\mathbf{p}_{0}, \mathbf{p}_{0} ; \epsilon\right)=1 \\
L\left(\mathbf{p}_{0}, \mathbf{p}_{0} ; \epsilon\right) & =-\int \frac{d \mathbf{k}}{(2 \pi)^{3}} \frac{u^{2}(k)}{\left(b+\frac{k^{2}}{2 \mu_{23}}\right)^{3}}
\end{aligned}
$$

The main singularity of the kernel of the integral equation (19) for the scattering wave function, which appears after removing the screening of the Coulomb interaction between charged particles, is caused by superposing the singularity of the Coulomb potential acting between the incident particle and the centre of mass of the two-body complex,

$$
V^{C}\left(\mathbf{p}-\mathbf{p}^{\prime}\right)=\frac{4 \pi e_{1} e_{2}}{\left(\mathbf{p}-\mathbf{p}^{\prime}\right)^{2}}
$$


that (according to Eqs. (33)) is contained in the kernel $U$, on the singularity of the free Green function

$$
\left\langle\mathbf{p}\left|g_{0}^{1}(\epsilon)\right| \mathbf{p}^{\prime}\right\rangle=(2 \pi)^{3} \delta\left(\mathbf{p}-\mathbf{p}^{\prime}\right)\left(\epsilon-\frac{p^{2}}{2 \mu_{1}}+i 0\right)^{-1} .
$$

The limiting passage to the non-screened Coulomb interaction in the equation (19) we perform after prior reformulation of the equation by extracting explicitly the term $g_{0}^{1}(\epsilon) \bar{V}^{C}$, which corresponds to the pure Coulomb interaction, from the kernel (19). Transposing this term to the left side of the equation (19) and acting by the inverted operator $1-g_{0}^{1}(\epsilon) \bar{V}^{C}$ on both of the sides of the obtained equation, we write the equation for the function $\bar{\chi}_{\mathbf{p}_{0}}$ in the following form

$$
\bar{\chi}_{\mathbf{p}_{0}}=\bar{\psi}_{\mathbf{p}_{0}}+\bar{g}^{C}(\epsilon)\left[\bar{U}(\epsilon) S\left(\epsilon-b-h_{0}^{1}\right)-\bar{V}^{C}\right] \bar{\chi}_{\mathbf{p}_{0}} .
$$

The introduced scattering function $\bar{\psi}_{\mathbf{p}_{0}}^{C}$ for the screened Coulomb potential $\bar{V}^{C}(32)$ and the corresponding Green function $\bar{g}^{C}(\epsilon)$ are determined by the equations:

$$
\begin{aligned}
\bar{\psi}_{\mathbf{p}_{0}}^{C} & =\varphi_{\mathbf{p}_{0}}+g_{0}^{1}(\epsilon) \bar{V}^{C} \bar{\psi}_{\mathbf{p}_{0}}^{C}, \\
\bar{g}^{C}(\epsilon) & =g_{0}^{1}(\epsilon)+g_{0}^{1}(\epsilon) \bar{V}^{C} \bar{g}^{C}(\epsilon) .
\end{aligned}
$$

The passage to the non-screened Coulomb interaction is performed in the regularized equation (39) automatically taking into account accordingly to Refs. $[5,6]$ that the functions $\bar{\psi}_{\mathbf{p}_{0}}^{C}$ and $\bar{\chi}_{\mathbf{p}_{0}}$ for $R_{s} \rightarrow \infty$ have a form of the products of the known singular phase factor and the respective functions $\psi_{\mathbf{p}_{0}}^{C}$ and $\chi_{\mathbf{p}_{0}}$, which present a finite limit and describe the physical scattering process with the nonscreened Coulomb interaction,

$$
\begin{aligned}
& \bar{\psi}_{\mathbf{p}_{0}}^{C}(\mathbf{p}) \stackrel{R_{s} \rightarrow \infty}{\longrightarrow} \exp \left[-i \eta\left(\ln 2 p_{0} R_{s}-\hat{\mathrm{C}}\right)\right] \psi_{\mathbf{p}_{0}}^{C}(\mathbf{p}), \\
& \bar{\chi}_{\mathbf{p}_{0}}^{C}(\mathbf{p}) \stackrel{R_{s} \rightarrow \infty}{\longrightarrow} \exp \left[-i \eta\left(\ln 2 p_{0} R_{s}-\hat{\mathrm{C}}\right)\right] \chi_{\mathbf{p}_{0}}^{C}(\mathbf{p}),
\end{aligned}
$$

where

$$
\eta=\frac{\mu_{1} e_{1} e_{2}}{\hbar^{2} p_{0}}=\frac{e_{1} e_{2}}{\hbar} \sqrt{\frac{\mu_{1}}{2 \epsilon}}
$$

is Sommerfeld's parameter, $\hat{\mathrm{C}}=0.577215 \ldots$ being Euler's constant [26].

The final integral equation for the function $\chi_{\mathbf{p}_{0}}^{C}(\mathbf{p})$ we obtain in the form

$$
\begin{aligned}
\chi_{\mathbf{p}_{0}}(\mathbf{p})= & \psi_{\mathbf{p}_{0}}^{C}(\mathbf{p})+\int \frac{d \mathbf{p}^{\prime}}{(2 \pi)^{3}} \int \frac{d \mathbf{p}^{\prime \prime}}{(2 \pi)^{3}}\left\langle\mathbf{p}\left|g^{C}(\epsilon)\right| \mathbf{p}^{\prime \prime}\right\rangle \\
& \cdot\left[U\left(\mathbf{p}^{\prime \prime}, \mathbf{p}^{\prime} ; \epsilon\right) S\left(\epsilon-b-\frac{p \prime^{2}}{2 \mu_{1}}\right)-V^{C}\left(\mathbf{p}^{\prime \prime}-\mathbf{p}^{\prime}\right)\right] \chi_{\mathbf{p}_{0}}\left(\mathbf{p}^{\prime}\right) .
\end{aligned}
$$


where $\psi_{\mathbf{p}_{0}}^{C}(\mathbf{p})$ is the Coulomb scattering wave function in the momentum space,

$$
\psi_{\mathbf{p}_{0}}^{C}(\mathbf{p})=C(\eta) \varphi_{\mathbf{p}_{0}}^{C}(\mathbf{p}), \quad \varphi_{\mathbf{p}_{0}}^{C}(\mathbf{p})=\int d \boldsymbol{\rho} e^{-i \mathbf{p} \boldsymbol{\rho}} \varphi_{\mathbf{p}_{0}^{C}}(\boldsymbol{\rho}) .
$$

In the configuration space the Coulomb scattering function $\psi_{\mathbf{p}_{0}}^{C}(\boldsymbol{\rho})$ has the form [24]

$$
\psi_{\mathbf{p}_{0}}^{C}(\boldsymbol{\rho})=C(\eta) \varphi_{\mathbf{p}_{0}}^{C}(\boldsymbol{\rho}), \quad \varphi_{\mathbf{p}_{0}}^{C}(\boldsymbol{\rho})=e^{i \mathbf{p}_{0} \boldsymbol{\rho}}{ }_{1} F_{1}\left(-i \eta, 1, i\left[p_{0} \rho-\mathbf{p}_{0} \boldsymbol{\rho}\right]\right)
$$

where $\boldsymbol{\rho} \equiv \boldsymbol{\rho}_{1}=\left(m_{2} \mathbf{r}_{2}+m_{3} \mathbf{r}_{3}\right) / m_{23}-\mathbf{r}_{1}$ is the relative Jacobi radius-vector between the centre of mass for the particles 2 and 3 and the particle 1 ( $\mathbf{r}_{i}$ being the radiusvector of the particle $i$ ), that corresponds to the relative momentum $\mathbf{p}_{1}(7)$. The coefficient

$$
C(\eta)=\exp \left(-\frac{1}{2} \pi \eta\right) \Gamma(1+i \eta)
$$

in Eqs. (44) and (45) corresponds to the normalization of the incident wave on the unit probability density

$$
\left|\psi_{\mathbf{p}_{0}}^{C}(\rho \rightarrow \infty)\right| \rightarrow 1,
$$

providing the known result for the probability that the particle is at the point $\rho=0$ (relatively to the probability that it is in the incident beam) in the case of the pure Coulomb scattering of two charges - the penetration factor for the Coulomb field of a point particle

$$
P_{0}=\frac{\left|\psi_{\mathbf{p}_{0}}^{C}(\rho=0)\right|^{2}}{\left|\psi_{\mathbf{p}_{0}}^{C}(\rho \rightarrow \infty)\right|^{2}}=|C(\eta)|^{2}=\frac{2 \pi \eta}{e^{2 \pi \eta}-1} .
$$

It is obvious that the pole and Coulomb singularities in the kernel of the equation (43), unlike Eq. (19), do not appear in one point and hence the integral equation (43) is polar.

The solution of the equation (43), which expresses $\chi_{\mathbf{p}_{0}}$ in terms $\psi_{\mathbf{p}_{0}}^{C}$, can be written in the following form:

$$
\chi_{\mathbf{p}_{0}}=\psi_{\mathbf{p}_{0}}^{C}+R(\epsilon) \psi_{\mathbf{p}_{0}}^{C},
$$

where $R(\epsilon)$ is the resolvent of the non-screened kernel $K(\epsilon)$,

$$
\begin{gathered}
R(\epsilon)=[1-K(\epsilon)]^{-1}-1, \\
K(\epsilon)=g^{C}(\epsilon)\left[U(\epsilon) S\left(\epsilon-b-h_{0}^{1}\right)-V^{C}\right] .
\end{gathered}
$$

The same behaviour as (41) when the screening distance tends to infinity $\left(R_{s} \rightarrow \infty\right)$ is also the case for the effective function $\bar{\psi}_{\mathbf{p}_{0}}^{\text {eff }}(24)$,

$$
\bar{\psi}_{\mathbf{p}_{0}}^{e f f}(\mathbf{p}) \stackrel{R_{s} \rightarrow \infty}{\longrightarrow} \exp \left[-i \eta\left(\ln 2 p_{0} R_{s}-\hat{\mathrm{C}}\right)\right] \psi_{\mathbf{p}_{0}}^{\text {eff }}(\mathbf{p})
$$


that follows immediately from the representation (25) of the function $\bar{\psi}_{\mathbf{p}_{0}}^{\text {eff }}$ in terms of the function $\bar{\chi}_{\mathbf{p}_{0}}^{e f f}$ and the behaviour (41) of the function $\bar{\chi}_{\mathbf{p}_{0}}^{e f f}$. Extracting from the expression (25) in the limit case $R_{s} \rightarrow \infty$ the singular phase factors, according to Eqs. (41) and (52), we obtain the expression for the effective scattering wave function $\psi_{\mathbf{p}_{0}}^{e f f}$, that describes the scattering process in the case of the non-screened potential

$$
\psi_{\mathbf{p}_{0}}^{e f f}(\mathbf{p})=\chi_{\mathbf{p}_{0}}(\vec{p})+\int \frac{d \mathbf{p}^{\prime}}{(2 \pi)^{3}} Z\left(\mathbf{p}, \mathbf{p}^{\prime} ; \epsilon\right) S\left(\epsilon-b-\frac{p \prime^{2}}{2 \mu_{1}}\right) \chi_{\mathbf{p}_{0}}\left(\mathbf{p}^{\prime}\right) .
$$

The formula (53) for the effective wave function can be written in the form of the expression through the Coulomb wave function $\psi_{\mathbf{p}_{0}}^{C}$ using the formula (49) for the function $\chi_{\mathbf{p}_{0}}$ (and the formula $(27)$ in the case of the non-screened kernel $Z$ ),

$$
\psi_{\mathbf{p}_{0}}^{e f f}=\psi_{\mathbf{p}_{0}}^{C}+g_{0}^{1}(\epsilon)\left\{W(\epsilon) S\left(\epsilon-b-h_{0}^{1}\right)[1+R(\epsilon)]-V^{C}\right\} \psi_{\mathbf{p}_{0}}^{C} .
$$

\section{The probability of the penetration of a charged particle through the Coulomb field of a two-particle complex}

In the context of the elaborated three-particle formalism, it is of interest to investigate the approach of a charged particle and a charged two-particle bound complex, specifically, the phenomenon of the penetration of a charged particle through the complicated Coulomb barrier of a two-particle complex.

In the configuration space, the effective wave function can be found using the Fourier transform

$$
\psi_{\mathbf{p}_{0}}^{\text {eff }}(\boldsymbol{\rho})=\int \frac{d \mathbf{p}}{(2 \pi)^{3}} i \mathbf{p} \boldsymbol{\rho}_{\psi_{\mathbf{p}_{0}}^{\text {eff }}(\mathbf{p})} .
$$

The value of the effective scattering wave function at the point of the configuration space $\rho=0$ can be determined according to Eq. (55) as an integral of the Fourier image over the whole momentum space,

$$
\psi_{\mathbf{p}_{0}}^{e f f}(\rho=0)=\int \frac{d \mathbf{p}}{(2 \pi)^{3}} \psi_{\mathbf{p}_{0}}^{e f f}(\mathbf{p}) .
$$

Using the expression (54) for $\psi_{\mathbf{p}_{0}}^{e f f}(\mathbf{p})$, the formula (56) is written in the form

$$
\begin{aligned}
\psi_{\mathbf{p}_{0}}^{e f f}(\rho=0)= & \psi_{\mathbf{p}_{0}}^{C}(\rho=0) \\
& +\int \frac{d \mathbf{p} d \mathbf{p}^{\prime}}{(2 \pi)^{6}} \frac{1}{\epsilon-\frac{p^{2}}{2 \mu_{1}}+i 0}\left\{\int \frac{d \mathbf{p}^{\prime \prime}}{(2 \pi)^{3}} W\left(\mathbf{p}, \mathbf{p}^{\prime \prime} ; \epsilon\right) S\left(-b+\epsilon_{p^{\prime \prime}}\right)\right. \\
& \left.\cdot\left\langle\mathbf{p}^{\prime \prime}|[1+R(\epsilon)]| \mathbf{p}^{\prime}\right\rangle-V^{C}\left(\mathbf{p}-\mathbf{p}^{\prime}\right)\right\} \psi_{\mathbf{p}_{0}}^{C}\left(\mathbf{p}^{\prime}\right) .
\end{aligned}
$$


The probability of finding a charge particle at the point of the centre of mass of a two-particle complex (relatively to the probability of finding it in the incident beam) is equal to

$$
P=\frac{\left|\psi_{\mathbf{p}_{0}}^{e f f}(\rho=0)\right|^{2}}{\left|\psi_{\mathbf{p}_{0}}^{e f f}(\rho \rightarrow \infty)\right|^{2}} .
$$

The quantity $P$ is referred to as the penetration factor for the Coulomb field of the two-particle complex.

In accordance with Eqs. (44) and (45), extracting from the expression (57) the common coefficient - the normalized factor of the Coulomb scattering function $C(\eta)$ - the formula (57) for $\psi_{\mathbf{p}_{0}}^{e f f}(\rho=0)$ can be given in the form

$$
\psi_{\mathbf{p}_{0}}^{\text {eff }}(\rho=0)=C(\eta)\left[1+D\left(\eta, p_{0}\right)\right],
$$

where the dimensionless quantity

$$
\begin{aligned}
D\left(\eta, p_{0}\right)= & \int \frac{d \mathbf{p} d \mathbf{p}^{\prime}}{(2 \pi)^{6}} \frac{1}{\epsilon_{p}}\left\{\int \frac{d \mathbf{p}^{\prime \prime}}{(2 \pi)^{3}} W\left(\mathbf{p}, \mathbf{p}^{\prime \prime} ; \epsilon\right) S\left(-b+\epsilon_{p^{\prime \prime}}\right)\right. \\
& \left.\cdot\left\langle\mathbf{p}^{\prime \prime}|[1+R(\epsilon)]| \mathbf{p}^{\prime}\right\rangle-V^{C}\left(\mathbf{p}-\mathbf{p}^{\prime}\right)\right\} \varphi_{\mathbf{p}_{0}}^{C}\left(\mathbf{p}^{\prime}\right),
\end{aligned}
$$

that describes the structure effect of the complex depends on the energy of the relative motion of the particle and the complex $\epsilon$, the parameter $\eta$ and two parameters which characterize the potential of the interaction between the constituents of the complex or the bound state of the complex, in capacity of those it is convenient to use the binding energy $b$ and a quantity connected with the range of the interaction.

Since the kernel of the reduced operator in parentheses of the expression (54) does not contain the operator of the long-range Coulomb interaction, the normalization of the effective scattering wave function $\psi_{\mathbf{p}_{0}}^{\text {eff }}$ at infinitely large distances does not differ from the corresponding normalization of the scattering Coulomb function (47),

$$
\left|\psi_{\mathbf{p}_{0}}^{e f f}(\rho \rightarrow \infty)\right| \rightarrow 1
$$

Substituting the formulae (59) and (61) into Eq. (58), the expression for the probability of the penetration of the incident charged particle into the centre of mass of the two-particle complex (or the penetration factor for the particle in the Coulomb field of the complex) is expressed in the form

$$
P=P_{0}\left\{1+2 \operatorname{Re} D+|D|^{2}\right\}
$$

where $P_{0}$ is the probability of finding at one point of the incident particle with the charge $e_{1}$ and the mass $m_{1}$ and a point "particle" with the charge and mass 
equal to the summary charge and mass of the particles of the complex, $e_{2}$ and $m_{23}$, respectively, and located at the centre of mass of the complex - the penetration factor for the charge particle in the Coulomb field of the structureless complex (see the formula (48)). The sum of the second and third terms in the parentheses of the expression (62) describes the relative deviation of the penetration factor for the charged particle in the Coulomb field of the two-particle complex from that of the corresponding point charge,

$$
\Delta \equiv \frac{P-P_{0}}{P_{0}}=2 \operatorname{Re} D+|D|^{2} .
$$

In a general way, characterizing an influence of the structure of the complex on the penetration of the point particle 1 through the Coulomb field of the complex, the quantity $\Delta$ (63) depends on the charges and masses of the particles of the system, the incident momentum $p_{0}$ and the parameters that describe the bound state of the two-body complex.

\subsection{The relative deviation $\Delta$ in the linear approximation in the parameter $\eta$}

Let us determine the probability of approaching a charge point particle and twoparticle complex ussing the formula (60) in the assumption that the parameter $|\eta|$ is small,

$$
|\eta|<1
$$

In the linear approximation in $\eta$, taking in the formula (60) in accordance with Eqs. (44) and (45) (along with Eqs. (33) and (34)) only the first terms of expansions in the parameter $\eta$ of the function $\varphi_{\vec{p}_{0}}^{C}$ and the kernels $W$ and $R$ into account, we have

$$
\begin{gathered}
\varphi_{\mathbf{p}_{0}}^{C}\left(\mathbf{p}^{\prime}\right)=\varphi_{\mathbf{p}_{0}}\left(\mathbf{p}^{\prime}\right)+O(\eta), \\
W\left(\mathbf{p}, \mathbf{p}_{0} ; \epsilon\right)=V^{C}\left(\mathbf{p}-\mathbf{p}_{0}\right) J\left(\mathbf{p}, \mathbf{p}_{0} ; \epsilon\right)+o(\eta), \quad J\left(\mathbf{p}, \mathbf{p}_{0} ; \epsilon\right)=F_{00}\left(\left|\mathbf{p}-\mathbf{p}_{0}\right|\right), \\
R\left(\mathbf{p}^{\prime \prime}, \mathbf{p}^{\prime} ; \epsilon\right)=(2 \pi)^{3} \delta\left(\mathbf{p}^{\prime \prime}-\mathbf{p}^{\prime}\right)+O(\eta),
\end{gathered}
$$

where $F_{00}(q)$ is the formfactor of the charge distribution of the two-particle complex (that is in a $S$-wave bound state),

$$
F_{00}(q)=\int \frac{d \mathbf{k}}{(2 \pi)^{3}} \psi_{0}^{*}(\mathbf{k}) \psi_{0}\left(\mathbf{k}-\frac{m_{3}}{m_{23}} \mathbf{q}\right)=\int d \mathbf{r} \exp \left(i \frac{m_{3}}{m_{23}} \mathbf{q r}\right)\left|\psi_{0}(\mathbf{r})\right|^{2},
$$

and the expression for the quantity $D$ is given by

$$
D\left(\eta, p_{0}\right)=8 \pi \eta p_{0} \int \frac{d \mathbf{p}}{(2 \pi)^{3}} \frac{1-F_{00}\left(\left|\mathbf{p}-\mathbf{p}_{0}\right|\right)}{\left(p^{2}-p_{0}^{2}-i 0\right)\left(\mathbf{p}-\mathbf{p}_{0}\right)^{2}}+o(\eta)
$$




$$
=\eta\left[A\left(p_{0}\right)+i B\left(p_{0}\right)\right]+o(\eta), \quad|\eta|<1 .
$$

Here the following notations are used:

$$
\begin{aligned}
& A\left(p_{0}\right)=\frac{1}{\pi} \int_{0}^{\infty} d q \frac{1-F_{00}(q)}{q} \ln \left(\frac{q+2 p_{0}}{\left|q-2 p_{0}\right|}\right) \\
& B\left(p_{0}\right)=\int_{0}^{2 p_{0}} d q \frac{1-F_{00}(q)}{q}
\end{aligned}
$$

or in terms of Eq. (66),

$$
\begin{aligned}
& A\left(p_{0}\right)=\frac{\pi}{2}-4 \int_{0}^{\infty} d r r^{2}\left|\psi_{0}(r)\right|^{2} \int_{0}^{\infty} \frac{d q}{q} j_{0}\left(\frac{m_{3}}{m_{23}} q r\right) \ln \left(\frac{q+2 p_{0}}{\left|q-2 p_{0}\right|}\right), \\
& \left.B\left(p_{0}\right)=4 \pi \int_{0}^{\infty} d r r^{2}\left|\psi_{0}(r)\right|^{2}\left[j_{(} x_{0}\right)+\operatorname{Cin}\left(x_{0}\right)-1\right], \quad x_{0}=2 \frac{m_{3}}{m_{23}} p_{0} r
\end{aligned}
$$

where $j_{0}(x)$ is the spherical Bessel function, $\operatorname{Cin}(x)$ is the integral function, $\operatorname{Cin}(x)=$ $-\mathrm{Ci}(x)+\ln (x)+\hat{\mathrm{C}}, \mathrm{Ci}(x)$ being the integral cosine, and $\hat{\mathrm{C}}$ is the Euler constant $[26]$.

In the limiting case of a negligible mass of the neutral constituent particle of the complex $\left(m_{3} \ll m_{2}\right)$, the centre of mass and the centre of charge are coincident $\left(m_{3} / m_{23} \rightarrow 0\right)$, the form factor approaches unity, $F_{00}(q) \rightarrow 1$, and the quantities $A\left(p_{0}\right)$ and $B\left(p_{0}\right)$ (as $\left.D\right)$ tend to zero.

Using Eq. (67), for the ratio of the probability of penetration of a charged particle to the centre of mass $P(62)$ to the probability of approaching a charged particle and a point charge (that equals to the charge of the complex) $P_{0}(48)$ we obtain

where the quantity

$$
\frac{P}{P_{0}}=1+\Delta
$$

$$
\Delta=2 A\left(p_{0}\right) \eta+o(\eta)
$$

describes the deviation of the probability $P$ from $P_{0}$.

For the separable potential (9) with the factor $u(k)$ of the Yukawa form,

$$
u(k)=\frac{\sqrt{2 \pi \kappa \beta(\kappa+\beta)^{3}}}{\mu_{23}} \frac{1}{k^{2}+\beta^{2}},
$$

where the parameter $\beta$ characterizes the inverse of the range of interaction and the normalization corresponds to the normalization of the wave function of the complex to unit (12), the form factor of the charge distribution of the two-body complex (66) can be written in the analytical form,

$$
\begin{aligned}
F_{00}(q)= & \frac{2 m_{23}}{m_{3}} \frac{\kappa \beta(\beta+\kappa)}{(\beta-\kappa)^{2} q}\left\{\arctan \left(\frac{1}{2} \frac{m_{3}}{m_{23}} \frac{q}{\kappa}\right)\right. \\
& \left.+\arctan \left(\frac{1}{2} \frac{m_{3}}{m_{23}} \frac{q}{\beta}\right)-2 \arctan \left(\frac{m_{3}}{m_{23}} \frac{q}{\beta+\kappa}\right)\right\} .
\end{aligned}
$$


Using Eq. (73), the expression for the quantity $A\left(p_{0}\right)(68)$ that is contained in Eq. (71), becomes

$$
\begin{aligned}
A\left(p_{0}\right)= & \frac{\pi}{2}-\frac{\kappa_{0} \beta_{0}\left(\beta_{0}+\kappa_{0}\right)}{\pi\left(\beta_{0}-\kappa_{0}\right)^{2}} \int_{0}^{\infty} \frac{d x}{x^{2}}\left[\arctan \left(\frac{x}{\kappa_{0}}\right)\right. \\
& \left.+\arctan \left(\frac{x}{\beta_{0}}\right)-2 \arctan \left(\frac{2 x}{\beta_{0}+\kappa_{0}}\right)\right] \ln \left(\frac{x+1}{|x-1|}\right),
\end{aligned}
$$

where

$$
\kappa_{0}=\frac{m_{23}}{m_{3}} \frac{\kappa}{p_{0}}, \quad \beta_{0}=\frac{m_{23}}{m_{3}} \frac{\beta}{p_{0}} .
$$

The quantity $A$ (68) depends on the quantities which describe the bound state of the two-particle complex — on the binding energy $b=\kappa^{2} / 2 \mu_{23}$ and the parameter $\beta$ for the potential with the factor $(72)$ - and also on the incident momentum of the relative motion of the particle and the centre of mass of the complex $p_{0}$ (or the energy $\left.\epsilon=p_{0}^{2} / 2 \mu_{1}\right)$. Acording to Eq. (74), it as dimensionless quantity is constructed from two dimensionless parameters, $\kappa_{0}$ and $\beta_{0}(75)$. In view of the fact that the expression (74) contains a linear combination of three like integrals, the quantity $A$ is written as

$$
\left.A_{(} p_{0}\right)=\frac{\pi}{2}-\frac{\kappa_{0} \beta_{0}\left(\beta_{0}+\kappa_{0}\right)}{\pi\left(\beta_{0}-\kappa_{0}\right)^{2}}\left[a\left(\frac{1}{\kappa_{0}}\right)+a\left(\frac{1}{\beta_{0}}\right)-2 a\left(\frac{2}{\kappa_{0}+\beta_{0}}\right)\right],
$$

where

$$
a_{(\xi)}=\int_{0}^{\infty} \frac{d x}{x^{2}} \arctan (\xi x) \ln \left(\frac{x+1}{|x-1|}\right) .
$$

We note that in the case of the potential (9), (72) the wave function of the complex in the bound state (12) having a physical behaviour at asymptotically large distances between the particles is possible only provided that the unequality

$$
\kappa<\beta
$$

is obeyed. It immediately follow the unequalities between the values of the argument of the function $a(\xi)$, which are contained in the expression (76):

$$
\frac{1}{\beta_{0}}<\frac{2}{\kappa_{0}+\beta_{0}}<\frac{1}{\kappa_{0}} .
$$

In a more compact form, simplifying the expression for linear combination of the arctangent functions with different arguments in Eq. (74) and taking into account the value of the integral

$$
\int_{0}^{\infty} \frac{d x}{x} \ln \frac{x+1}{|x-1|}=\frac{\pi^{2}}{2}
$$


the formula (74) can be written as

$$
A\left(p_{0}\right)=\frac{2}{\pi} \eta \int_{0}^{\infty} \frac{d x}{x^{2}} \ln \left(\frac{x+1}{|x-1|}\right) \Phi\left(x, p_{0}\right),
$$

where

$$
\begin{gathered}
\Phi\left(x, p_{0}\right)=x-\frac{d}{f} \arctan \left(\frac{f x}{4 x^{4}+c x^{2}+d}\right) \\
f=\left(\beta_{0}+\kappa_{0}\right)\left(\beta_{0}-\kappa_{0}\right)^{2}, \quad c=3 \beta_{0}^{2}+2 \beta_{0} \kappa_{0}+3 \kappa_{0}^{2}, \quad d=\beta_{0} \kappa_{0}\left(\beta_{0}+\kappa_{0}\right)^{2} .
\end{gathered}
$$

From the formulae (81) and (82) it follows at once that the function $A\left(p_{0}\right)$ is positive for all positive values of the argument, since the function $\Phi\left(x, p_{0}\right)$ and the integrand in Eq. (81) take positive values for all $x>0$ (at the point $x=0$ the function $\Phi$ and its first and second derivatives $\Phi^{\prime}$ and $\Phi^{\prime \prime}$ are vanished).

As a consequence, in the first approximation of the expansion of $P / P_{0}$ in $\eta(70)$, the correction for the structure of the complex $\Delta(71)$ is positive $(\Delta>0)$ in the case of the repulsive Coulomb interaction between charged particles $\left(e_{1} e_{2}>0\right)$ and negative $(\Delta<0)$ in the case of the attractive Coulomb interaction $\left(e_{1} e_{2}<0\right)$. Hence, allowance for the structure of complex results in the penetration factor to increase for like charges of the particle and the complex whereas to decrease for unlike charges,

$$
P>P_{0}, \quad e_{1} e_{2}>0, \quad P<P_{0}, \quad e_{1} e_{2}<0
$$

In this approximation, the correction for the structure of the complex $\Delta$ is proportional to the reduced mass of the incident particle and the particles of the complex $\mu_{1}$ (the inverse Bohr radius of an atom composed of the incident particle and the two-particle complex).

\subsection{A simple analytical estimation of $\Delta$ in a restricted range of small values of $p_{0}$}

The magnitude of the influence of the structure of the two-fragment complex on the probability of the penetration of a charged particle through the Coulomb field of the complex can be easily estimated in the event that the incident momentum $p_{0}$ is small relative to $\kappa$ such that

$$
\frac{1}{\kappa_{0}}=\frac{m_{3}}{m_{23}} \frac{p_{0}}{\kappa}<1 .
$$

Then it becomes possible to perform an approximate integration in Eq. (76) in an explicit form. We restrict ourselves to taking into account the first four terms in the 
expansion of the function $a(\xi)(76)$ in the power series in $\xi$,

$$
a(\xi)=a(0)+\xi a^{\prime}(0)+\frac{1}{2} \xi^{2} a^{\prime \prime}(0)+o\left(\xi^{2}\right),
$$

Splitting the interval of the integration in the expressions for the function $a(\xi)(76)$ and its derivatives into two parts - from 0 to 1 and from 1 to $\infty$ - and performing the integration with the help of Eq. (80) and the formulae [27]

$$
\begin{aligned}
\int_{0}^{1} d x x \ln \left(\frac{1+x}{1-x}\right)=1, & \int_{0}^{1} d x \frac{x}{x^{2}+\xi^{2}} \ln \left(\frac{1+x}{1-x}\right)=\left[\arctan \left(\frac{1}{\xi}\right)\right]^{2}, \\
\int_{0}^{1} d x \frac{x}{\left(x^{2}+\xi^{2}\right)^{2}} \ln \left(\frac{1+x}{1-x}\right) & =\frac{1}{\xi\left(1+\xi^{2}\right)} \arctan \left(\frac{1}{\xi}\right), \\
\int_{0}^{1} d x \frac{x}{\left(x^{2}+\xi^{2}\right)^{3}} \ln \left(\frac{1+x}{1-x}\right) & =\frac{1}{4 \xi^{3}\left(1+\xi^{2}\right)^{3}}\left[\left(1+3 \xi^{2}\right) \arctan \left(\frac{1}{\xi}\right)+\xi\right] \\
\int_{0}^{1} d x \frac{x^{3}}{\left(x^{2}+\xi^{2}\right)^{3}} \ln \left(\frac{1+x}{1-x}\right) & =\frac{1}{4 \xi\left(1+\xi^{2}\right)^{2}}\left[\left(3+\xi^{2}\right) \arctan \left(\frac{1}{\xi}\right)-\xi\right]
\end{aligned}
$$

we then find the coefficients of the expansion (85):

$$
a(0)=0, \quad a^{\prime}(0)=\frac{\pi^{2}}{2}, \quad a^{\prime \prime}(0)=-\pi, \quad a^{\prime \prime \prime}(0)=0 .
$$

As a result, the approximated expression for the function $a(\xi)$ (85) takes the form

$$
a(\xi)=\frac{\pi^{2}}{2} \xi-\frac{\pi}{2} \xi^{2}+O\left(\xi^{3}\right), \quad 0<\xi<1 .
$$

In the formula (88) the expansion of the function $a(\xi)(76)$ is performed in the interval of convergence $0<\xi<1$. It is worth noting that the obtained expression (88) does not contradict to the odd parity of the function $a(\xi)(76)$, since the second derivative of the function $a^{\prime \prime}(\xi)$ has a finite discontinuity at the point $\xi=0$,

$$
a^{\prime \prime}(\xi) \longrightarrow-\operatorname{sgn}(\xi) \pi, \text { when } \xi \rightarrow 0
$$

taking the values of opposite signs when approaching 0 from the positive and negative values of the argument $\xi, a^{\prime \prime}\left(0_{ \pm}\right)=\mp \pi$. (Here, $\operatorname{sgn}(\xi)$ is the sign function of $\xi$.)

Substituting to Eq. (76) the first terms of the expansion of the function $a(\xi)$ for all three values of the argument in Eq. (76), which in accordance to the inequalities (79) and (84) are smaller than 1 , we wright the main (linear with respect to $p_{0}$ ) term of the expansion of the quantity $A$ in the form

$$
\begin{aligned}
A\left(p_{0}\right) & =\frac{1}{2}\left(\frac{1}{\kappa_{0}}+\frac{2}{\kappa_{0}+\beta_{0}}+\frac{1}{\beta_{0}}\right)+o\left(\frac{1}{\kappa_{0}}\right) \\
& =\frac{1}{2} \frac{m_{3}}{m_{23}} \frac{p_{0}}{\kappa} f(\beta, \kappa)+o\left(\frac{1}{\kappa_{0}}\right)
\end{aligned}
$$


where

$$
f(\beta, \kappa)=\frac{\beta^{2}+4 \beta \kappa+\kappa^{2}}{\beta(\beta+\kappa)},
$$

Introducing the mean radius of the two-particle complex in the considered case of the interaction $(9),(72)$,

$$
R=\frac{1}{2}\left\langle\psi_{0}|r| \psi_{0}\right\rangle=\frac{1}{4 \kappa} f(\kappa, \beta),
$$

we rewrite Eq. (89) as

$$
A\left(p_{0}\right)=\frac{2 m_{3}}{m_{23}} R p_{0}+o\left(\frac{1}{\kappa_{0}^{2}}\right) .
$$

Notice that the expression (89) immediately follows also from the formula for $A\left(p_{0}\right)$ (69), if in it to apply the expansion of the spherical Bessel function in small values of the argument, that is equivalent to the expansion (88) of the function $a(\xi)$ in Eq. (76) in small $\xi$.

In the employed approximation, the relative deviation of the penetration factor for the Coulomb field of the two-particle complex $P$ from the penetration factor for the Coulomb field of the corresponding point charge $P_{0}(74)$ does not depend on the incident momentum $p_{0}$,

$$
\Delta=\frac{m_{1} m_{3}}{M} \frac{e_{1} e_{2}}{\hbar^{2} \kappa} f(\kappa, \beta)=4 \frac{m_{1} m_{3}}{M} \frac{e_{1} e_{2}}{\hbar^{2}} R,
$$

turning out to be proportional to the ratio of the mean radius of the two-particle complex to Bohr's radius of an atom composed of the incident particle and the complex, $a_{B} \equiv \hbar^{2} / \mu_{1}\left|e_{1} e_{2}\right|$,

$$
\Delta=\operatorname{sgn}\left(e_{1} e_{2}\right) 4 \frac{m_{3}}{m_{23}} \frac{R}{a_{B}} .
$$

In the limiting case of the zero-range interaction between the constituent particles 2 and 3 of bound complex that follows from the model of the separable potential (9), (72) when the parameter $\beta$ becomes infinitely large $(\beta \rightarrow \infty)$, the factor $(90)$ tends to $1, f(\kappa, \beta) \rightarrow 1, R \rightarrow 1 / 4 \kappa$, and the correction for structure $\Delta$ takes the form

$$
\Delta \rightarrow \Delta_{z r}=\frac{m_{1} m_{3}}{M} \frac{e_{1} e_{2}}{\hbar^{2} \kappa}
$$

The interval of applicability of the formula (93) is limited by the values of the relative incident momentum $p_{0}$ that satisfy simultaneously to the conditions (64) and (84), providing the convergence of the expansions in series of the function $\Delta(\eta)$ in the parameter $\eta(42)$ and the function $a\left(\frac{1}{\kappa_{0}}\right)$ in the parameter $\frac{1}{\kappa_{0}}(75)$, and is determined by the inequalities

$$
\frac{1}{a_{B}}<p_{0}<\frac{m_{23}}{m_{3}} \kappa
$$


The associated values of the energy $\epsilon=p_{0}^{2} / 2 \mu_{1}$ are limited by the inequalities

$$
\epsilon_{R}<\epsilon<\frac{m_{2} M}{m_{1} m_{3}} b
$$

where the lower boundary is Rydberg's energy for an atom of the charged incident particle 1 and the two-particle complex, $\epsilon_{R}=\mu_{1}\left|e_{1} e_{2}\right|^{2} / 2 \hbar^{2}$, and the upper bound is proportional to the binding energy of the complex $b$.

\section{Numerical results and discussion}

Applying the above-described formalism by the use of the formulae (71), (81) and (82)), we have performed calculations of the deviation of the relative penetration factor, $\Delta$, in specific cases of collision between a charged incident particle of various mass (the muon, the pion, the kaon and the proton) and the deuteron and between the proton and the lightest hypernuclei, ${ }_{\Lambda}^{3} \mathrm{H}$ and ${ }_{\Lambda}^{5} \mathrm{He}$, which have a well-marked two-fragment structure.

To describe the deuteron (as a system of the proton and the neutron in the bound $S$-state), the following values of the parameters of the potential (9),(72) $\kappa \equiv \kappa_{p n}$ and $\beta \equiv \beta_{p n}$ that correspond to the experimental values of the deuteron binding energy $b \equiv b_{p n}=2.224575(9) \mathrm{V}[28]$ and the triplet neutron-proton scattering length $a_{n p}^{t}=5.424(3) \mathrm{fm}[29]$ were used

$$
\kappa_{p n}=0.23161 \mathrm{fm}^{-1}, \beta_{p n}=1.3906 \mathrm{fm}^{-1} .
$$

In a similar way we fit the parameters of the potentials of the form (9), (72) that support the hypernuclei ${ }_{\Lambda}^{3} \mathrm{H}$ and ${ }_{\Lambda}^{5} \mathrm{He}$. The parameters $\kappa \equiv \kappa_{d \Lambda}$ and $\beta \equiv \beta_{d \Lambda}$ providing the existence of the bound S-state of the hypertriton ${ }_{\Lambda}^{3} \mathrm{H}$ as a two-fragment system that consists of the deuteron and the $\Lambda$-hyperon, we find based on the experimental value of the binding energy of the hypertriton, $b \equiv b_{d \Lambda}=0.13(5)$ $\mathrm{MeV}[30]$, and the value of the doublet $\Lambda$-hyperon-deuteron scattering length $a_{d \Lambda}^{d}$ $=15.9 \mathrm{fm}$. The latter follows from the correlation dependence $a_{d \Lambda}^{d}$ on $b_{d \Lambda}$ that has been calculated in the paper [31] (see also Ref. [32]) on the basis of the threeparticle description of the hypertriton as a system of the proton, the neutron and the $\Lambda$-hyperon with the use of the known experimental low-energy data on $\mathrm{p}$ - $\mathrm{n}$ and $\Lambda$-nucleon interaction. We find in this case

$$
\kappa_{d \Lambda}=0.06834 \mathrm{fm}^{-1}, \beta_{d \Lambda}=1.1938 \mathrm{fm}^{-1}
$$

For the hypernucleus ${ }_{\Lambda}^{5} \mathrm{He}$ as a two-fragment system composed of the $\alpha$-particle and $\Lambda$-hyperon, we find the values of the parameter $\kappa \equiv \kappa_{\alpha \Lambda}$ that corresponds to 
the experimental binding energy of the nucleus ${ }_{\Lambda}^{5} \mathrm{He}, b=b_{\alpha \Lambda}=3.12(2) \mathrm{MeV}$ [30], and the parameter $\beta \equiv \beta_{\alpha \Lambda}$, that (together with the experimental $b_{\alpha \Lambda}$ ) reproduces the value of the root-mean-square radius of the bound system ${ }_{\Lambda}^{5} \mathrm{He},\left\langle r^{2}\right\rangle_{\alpha \Lambda}^{1 / 2}=2.43$ $\mathrm{fm}$, calculated with the use of a potential model of $\alpha-\Lambda$ interaction proposed in the paper [33],

$$
\kappa_{\alpha \Lambda}=0.37094 \mathrm{fm}^{-1}, \beta_{\alpha \Lambda}=2.177 \mathrm{fm}^{-1} .
$$

The results of our calculation of the function $A\left(p_{0}\right)$, which characterizes the structure of each one of the considered two-fragment nuclear systems, the deuteron and two hypernuclei, ${ }_{\Lambda}^{3} \mathrm{H}$ and ${ }_{\Lambda}^{5} \mathrm{He}$, are shown in Fig.1. The solid lines in the figure are obtained according to the formulae (81) and (82), which correspond to the separable potential of interaction between fragments of the nuclear complex (9), (72) with the parameters (98)-(100), while the dotted lines are obtained for the zero-range interaction $(\beta \rightarrow \infty)$. It follows from the definition $(76),(77)$ that the function $A\left(p_{0}\right)$ rises as the incident momentum $p_{0}$ increases. The value of $A\left(p_{0}\right)$ depends essentially on the binding energy of the two-fragment system, increasing when going to a system with a lesser binding energy. Hence, the curve $A\left(p_{0}\right)$ for the deuteron is shifted up relative to the curve for the nucleus ${ }_{\Lambda}^{5} \mathrm{He}$, as does the curve for the nucleus ${ }_{\Lambda}^{3} \mathrm{H}$ relative to the curve for the deuteron. From the side of small values of $p_{0}$ in the range (96), the behaviour of $A\left(p_{0}\right)$ appears to be near linear in accordance with the result of our approximate estimate (92), the slope of the straght line being proportional to the radius of the corresponding two-fragment complex $R$. For each nuclear complex the curve $A\left(p_{0}\right)$ calculated with the finite-range interaction is shifted up in comparison of that calculated with the zero-range interaction.

In the Table 1 and the Figures 2-4, we present our numerical results for the relative deviation of the penetration factor for the Coulomb field of the two-fragment nucleus from the penetration factor for the Coulomb field of the corresponding point charge, $\Delta$, in relation to the incident momentum $p_{0}$, which have been obtained by using the expressions $(71),(81)$ and $(82)^{2}$. The calculations have been performed for the charged incident particle of various mass (the muon, the pion, the kaon and the proton) colliding with the deuteron and for the proton colliding with the hypernuclei ${ }_{\Lambda}^{3} \mathrm{H}$ and ${ }_{\Lambda}^{5} \mathrm{He}$.

The obtained values of $\Delta$ for $\mu-\mathrm{d}, \pi-\mathrm{d}, \mathrm{K}-\mathrm{d}$ and $\mathrm{p}-\mathrm{d}$ interactions differ from each other, since, to begin with, the incident particles have different masses, $m_{1}$, in this case $\Delta$ increases with increasing $\mu_{1}$. Also, as the data of the Table 1 and the Fig. 4 indicate, the quantity $\Delta$ depends essentially on the binding energy of

\footnotetext{
${ }^{2}$ Note that the data for $\Delta$ reported in the table of Ref. [34] are erroneous (except the results in the last line of the table). The corresponding corrected results are given in Table 1 of this paper.
} 
the two-fragment nucleus $b$ increasing with decreasing $b$. Comparing the results for $\mathrm{p}-\mathrm{d}, \mathrm{p}-{ }_{\Lambda}^{3} \mathrm{H}$ and $\mathrm{p}-{ }_{\Lambda}^{5} \mathrm{He}$ interactions between themselves, it should be remembered that in this case an additional doubling $\Delta$ for $\mathrm{p}-{ }_{\Lambda}^{5}$ He caused by that the charged fragment of the hypernucleus ${ }_{\Lambda}^{5} \mathrm{He}$ (the $\alpha$-particle ${ }_{2}^{4} \mathrm{He}$ ) has its charge twice as large as the charged fragments of the deuteron or the hypertriton.

For comparison, in the Table 2 we give the values of the relative deviation $\Delta$ calculated in the simple approximation (93) which is independent of the incident momentum $p_{0}$ and may be applied for values of the momentum $p_{0}$ in the range (96). The result for $\Delta$ in the explicit analytical form (93) is obtained by approximate integration in the formula (76) using the expansion of the function (77) in the powers of $\xi(88)$ with the parameter $\xi$ taking the values

$$
\frac{m_{3}}{m_{23}} \frac{p_{0}}{\kappa}, \quad 2 \frac{m_{3}}{m_{23}} \frac{p_{0}}{\kappa+\beta}, \quad \text { or } \frac{m_{3}}{m_{23}} \frac{p_{0}}{\beta} \text {. }
$$

The greater the binding energy of the two-fragment system, the more it is accurate the approximate integration and the wider it is the range of values of the momentum $p_{0}$ for which this approximation is valid. This is evident comparing the data in the Table 2 with the data of the direct numerical integration listed in the Table 1: the mentioned approximation is appropriate in the widest range of $p_{0}$ for the most strongly bounded two-fragment nucleus ${ }_{\Lambda}^{5} \mathrm{He}$.

Notice that the calculation data obtained in this work embrace energies both below and above the Coulomb barrier of the electrostatic repulsion energy between the positively charged projectile and nucleus $\epsilon^{b}=\left|e_{1} e_{2}\right| / R$. (The values of the momentum $p_{0}^{b}$ that correspond to the respective energies $\epsilon^{b} \equiv\left(p_{0}^{b}\right)^{2} / 2 \mu_{1}$ are given in the Table 2).

\section{Summary}

This paper is devoted to formulation of a consistent three-body approach to the description of the phenomenon of the penetration of a charged particle through the Coulomb field of a two-fragment (nuclear) complex consisted of one charged and one neutral particles in a bound state. The corresponding formalism has been constructed on the basis of the use of the three-body Faddeev method and the Watson-Feshbach technique for introducing the effective (optical) potential of the interaction between a particle and a bound system.

In view of the presence of the long-range Coulomb interaction, the corresponding Faddeev integral equations are non-Fredholm above the threshold of the elastic particle-complex scattering, in this connection our study starts with the use of the 
screened Coulomb interaction. The passage to the limit of the non-screened Coulomb potential and the regularization of the obtained equations have been performed in accordance with the Gorshkov-Vesselova recipe by way of the explicit isolation and removal of the known singular exponential Coulomb factor.

The three-body formalism that describes the penetration of the charged particle through the Coulomb field of a two-particle complex has been worked out in the general case for both attractive and repulsive fields. The formula for the relative deviation $\Delta$ of the penetration factor for the Coulomb field of the two-particle complex from the penetration factor for the Coulomb field of the corresponding point charge has been derived.

As a first step to the application of the developed formalism, we have derived a simple analytic expression for the relative deviation $\Delta$ by approximation the function $D\left(\eta, p_{0}\right)$ with the linear term of the expansion of $D$ as a power series in $\eta$, the Sommerfeld parameter for the Coulomb interaction between the particle and the centre of mass of the complex, when $|\eta|<1$. In this case, the correction for the structure of the complex, $\Delta$, was found to be positive for the repulsive Coulomb interaction between the charged particle and negative for the attractive Coulomb interaction. By way of illustration, the influence of the nucleus structure on the probability of the coming together of a charged particle (the muon, the pion, the kaon and the proton) and a two-fragment nucleus (the deuteron and the $\Lambda$-hypernuclei ${ }_{\Lambda}^{3} \mathrm{H}$ and ${ }_{\Lambda}^{5} \mathrm{He}$ ) has been studied. The calculated values of the relative deviation $\Delta$ (by the formulae (71),(81) and (82)) are given in the Table 1 and shown in the Figures $2-4$. The quantity $\Delta$ is proportional to the mass of the incident particle $m_{1}$ and to the charges of the incident particle and complex, $e_{1}$ and $e_{2}$, it depends essentially on the binding energy of the complex $b$, increasing with decreasing $b$. It has been found that the value of the correction for the structure, $\Delta$, calculated according to the model of the finite-range nuclear interaction exceeds noticeably the value of the corresponding correction for the structure $\Delta_{z r}$, calculated with the use of the model of the zero-range interaction, $|\Delta|>\left|\Delta_{z r}\right|$ (see the data of the Tables 1 and 2).

A simple estimation of the influence of the two-fragment complex on the probability of the penetration of a charged particle through the Gamow field of the complex has been made for a range of small values of the incident momentum $p_{0}$ (relative to the momentum $\kappa$ that corresponds to the binding energy of the twobody complex, b). By expanding the function $a(\xi)$ in Eq. (76) in the parameter $\xi$ with the proviso that $\xi<1$ and taking the first four terms of the expansion into consideration, the function $\Delta\left(p_{0}\right)$ has been approximated by the expression (94). In this approximation, the function $\Delta\left(p_{0}\right)$ appears to be independent of $p_{0}$ and the 
magnitude of $\Delta$ is determined by the ratio of the mean radius of the two-particle complex $R$ to the Bohr radius of an atom composing of the incident particle and complex, $a_{B}$.

The three-body formalism given in this paper can be generalized to other cases when both of fragments of the complex are charged and thus applied to research the penetration of charged particles through the Coulomb fields of nuclear, atomic and molecular systems. 
1 G.A. Gamow, Z. Phys. 51 (1928) 204; 52 (1928) 510.

2 E.U. Condon and R.W. Gurney, Phys. Rev. 33 (1929) 127.

3 S.P. Merkuriev and L.D. Faddeev, Quantum Theory of Scattering for FewBody Systems (Nauka, Moscow, 1985).

4 L.D. Faddeev, Zh. Eksp. Teor. Fiz. 39 (1960) 1459.

5 V.G. Gorshkov, Zh. Eksp. Teor. Fiz. 40 (1961) 1481.

6 A.M. Vesselova, Teor. Mat. Fiz. 3 (1970) 326.

7 V.F. Kharchenko and S.A. Storozhenko, Integral equations for three-nucleon problem with Coulomb interaction. Proton-deuteron scattering, Preprint ITP75-53E (Institute for Theoretical Physics, Kyiv, 1975).

8 E.O. Alt, P. Grassberger and W. Sandhas, Nucl. Phys. B 2 (1967) 167.

9 E.O. Alt, W. Sandhas, H. Zankel and H. Ziegelmann, Phys. Rev. Lett. 37 (1976) 1537.

10 E.O. Alt, W. Sandhas, H. Ziegelmann, Phys. Rev. C 17 (1978) 1981.

11 E.O. Alt, in Dynamics of Few Body Systems, eds. Gy. Bencze, P. Doleschall and J. Révai (KFKI, Budapest, 1986) p. 367.

12 V.F. Kharchenko, M.A. Navrotsky and S.A. Shadchin, Nucl. Phys. A 512 (1990) 294.

13 V.F. Kharchenko, M.A. Navrotsky and P.A. Katerinchuk, Nucl. Phys. A 552 (1993) 378.

14 C.R. Chen, G.L. Payne, J.L. Friar and B.F. Gibson, Rhys. Rev. C 39 (1989) 1261; C 44 (1991) 50.

15 V.F. Kharchenko and S.A. Shadchin, Few Body Systems 6 (1989) 45.

16 V.F. Kharchenko and S.A. Shadchin, Three-body theory of the effective interaction between a particle and a two-particle bound system, Preprint ITP93-24E (Institute for Theoretical Physics, Kyiv, 1993).

17 V.F. Kharchenko and S.O. Shadchin, Ukrainian J. Phys. 42 (1997) 912.

18 E.O. Alt and A.M. Mukhamedzhanov, Phys. Rev. A 51 (1995) 3852. 
19 V.F. Kharchenko, Ukrainian J. Phys. 45 (2000) 616.

20 V.F. Kharchenko, J. Phys. Studies 4 (2000) 245.

21 J.V. Noble, Phys. Rev. 161 (1967) 945.

22 N.C. Francis and K.M. Watson, Phys.Rev. 92 (1953) 291.

23 H. Feshbach, Ann. Phys. (N.Y.) 5 (1958) 357; 19 (1962) 287.

24 C.J. Joachain, Quantum Collision Theory (North-Holland - American Elsevier, Amsterdam - New-York, 1975).

25 V.F. Kharchenko and S.O. Shadchin, Ukrainian J. Phys. 42 (1997) 11.

26 Handbook of Mathematical Functions, eds. M.Abramowitz and I.A. Stegun (National Bureau of Standards, Applied Mathematics Series-55, 1964).

27 I.S. Gradshtein and I.M. Ryzhik, Tables of Integrals, Sums, Series and Products (Gos. Izd. Fiz. Mat. Lit., Moscow, 1962).

28 . van der Leun and C. Alderliesten, Nucl. Phys. A 380 (1982) 261.

29 L. Koester, W. Nistler, Z. Phys. A 272 (1975) 189.

30 M. Jurić et al., Nucl. Phys. B 52 (1973) 1.

31 V.V. Peresypkin and N.M. Petrov, Binding energy of hypertriton and doublet scattering length of $\Lambda$-hyperon-deuteron scattering for nonlocal separable potentials (Institute for Theoretical Physics, Preprint ITF-75-39R, Kyiv, $1975)$.

32 N.M. Petrov, Yad. Fiz. 48 (1988) 50.

33 Y. Kurihara, Y. Akaishi and H. Tanaka, Phys. Rev. C 31 (1985) 971.

34 V.F. Kharchenko and A.V. Kharchenko, Ukrainian J. Phys. 48 (2003) 775. 


\section{Figure captions}

Fig. 1. The quantity $A$ as a function of the incident momentum $p_{0}$ for the deuteron and two lambda hypernuclei, ${ }_{\Lambda}^{3} \mathrm{H}$ and ${ }_{\Lambda}^{5} \mathrm{He}$, calculated by the formulae (81) and (82). The solid curves correspond to the separable potential model (9) and (72) with the parameters $(98)-(100)$, the dotted curves - to the zero-range interaction $(\beta \rightarrow \infty)$.

Fig. 2. The deviation $\Delta$ versus $p_{0}$ for $\mu^{+}-\mathrm{d}$ and $\pi^{+}-\mathrm{d}$ scattering calculated by the formulae (81) and (82) that correspond to the separable neutron-proton interaction potential (9) and (72) with the parameters (98).

Fig. 3. The deviation $\Delta$ versus $p_{0}$ for $\mathrm{K}^{+}-\mathrm{d}$ scattering calculated by the formulae (81) and (82) that correspond to the separable neutron-proton interaction potential (9) and (72) with the parameters (98).

Fig. 4. The deviation $\Delta$ versus $p_{0}$ for $\mathrm{p}-\mathrm{d}$ (solid line), $\mathrm{p}-{ }_{\Lambda}^{3} \mathrm{H}$ (dashed line)

and $\mathrm{p}-{ }_{\Lambda}^{5} \mathrm{He}$ (dotted line) scattering calculated by the formulae (81) and (82) that correspond to the separable $\mathrm{n}-\mathrm{p}, \Lambda-\mathrm{t}$ and $\Lambda-\alpha$ interaction potential (9) and (72) with the parameters $(98)-(100)$. 
Table 1:

Values of the relative deviation $\Delta$ calculated for the separable potential model (9) and (72) by the formulae $(71),(81)$ and (82). The numbers in curly brackets are the values of the relative deviation for the zero-range interaction $(\beta \rightarrow \infty), \Delta_{z r}$

\begin{tabular}{|c|c|c|c|c|c|c|}
\hline$p_{0}, \mathrm{fm}^{-1}$ & $\mu^{ \pm}-\mathrm{d}$ & $\pi^{ \pm}-\mathrm{d}$ & $K^{ \pm}-d$ & $p-d$ & $\mathrm{p}-{ }_{\Lambda}^{3} \mathrm{H}$ & $\mathrm{p}-{ }_{\Lambda}^{5} \mathrm{He}$ \\
\hline \multirow[t]{2}{*}{0.01} & \pm 0.01160 & \pm 0.01507 & & & & \\
\hline & $\{ \pm 0.00799\}$ & $\{ \pm 0.01038\}$ & & & & \\
\hline \multirow[t]{2}{*}{0.05} & \pm 0.01158 & \pm 0.01504 & \pm 0.04525 & & & \\
\hline & $\{ \pm 0.00798\}$ & $\{ \pm 0.01036\}$ & $\{ \pm 0.03117\}$ & & & \\
\hline \multirow[t]{2}{*}{0.10} & \pm 0.01150 & \pm 0.01494 & \pm 0.04496 & 0.07195 & 0.16037 & 0.05272 \\
\hline & $\{ \pm 0.00793\}$ & $\{ \pm 0.01030\}$ & $\{ \pm 0.03099\}$ & $\{0.04960\}$ & $\{0.13772\}$ & $\{0.03608\}$ \\
\hline \multirow[t]{2}{*}{0.30} & \pm 0.01081 & \pm 0.01403 & \pm 0.04223 & 0.06759 & 0.12737 & 0.05242 \\
\hline & $\{ \pm 0.00751\}$ & $\{ \pm 0.00975\}$ & $\{ \pm 0.02935\}$ & $\{0.04697\}$ & $\{0.10997\}$ & $\{0.03590\}$ \\
\hline \multirow[t]{2}{*}{0.50} & \pm 0.00979 & \pm 0.01272 & \pm 0.03828 & 0.06126 & 0.10072 & 0.05183 \\
\hline & $\{ \pm 0.00690\}$ & $\{ \pm 0.00896\}$ & $\{ \pm 0.02695\}$ & $\{0.04313\}$ & $\{0.08754\}$ & $\{0.03554\}$ \\
\hline \multirow[t]{2}{*}{0.70} & & & \pm 0.03428 & 0.05486 & 0.08240 & 0.05099 \\
\hline & & & $\{ \pm 0.02450\}$ & $\{0.03921\}$ & $\{0.07209\}$ & $\{0.03504\}$ \\
\hline \multirow[t]{2}{*}{1.00} & & & \pm 0.02907 & 0.04653 & 0.06433 & 0.04938 \\
\hline & & & $\{ \pm 0.02127\}$ & $\{0.03405\}$ & $\{0.05682\}$ & $\{0.03408\}$ \\
\hline \multirow[t]{2}{*}{2.00} & & & \pm 0.01846 & 0.02954 & 0.03673 & 0.04271 \\
\hline & & & $\{ \pm 0.01444\}$ & $\{0.02311\}$ & $\{0.03328\}$ & $\{0.03007\}$ \\
\hline \multirow[t]{2}{*}{3.00} & & & & 0.02125 & 0.02557 & 0.03637 \\
\hline & & & & $\{0.01739\}$ & $\{0.02358\}$ & $\{0.02621\}$ \\
\hline \multirow[t]{2}{*}{4.00} & & & & 0.01652 & 0.01958 & 0.03121 \\
\hline & & & & $\{0.01394\}$ & $\{0.01827\}$ & $\{0.02301\}$ \\
\hline
\end{tabular}


Table 2:

Values of the relative deviation $\Delta$ (for the separable potential (9) and (72)) and $\Delta_{z r}$ (for the zero-range interaction $(\beta \rightarrow \infty)$ ) calculated by the approximate formula (93). The lower and upper bounds of the interval (96) of the values of the incident momentum $p_{0}$ for which the formula (93) is applicable, $1 / a_{B}$ and $\left(m_{23} / m_{3}\right) \kappa$, are given in $\mathrm{fm}^{-1}$. The momentum $p_{0}^{b}$ corresponds to the Coulomb barrier energy $\epsilon^{b}=\left|e_{1} e_{2}\right| / R$

\begin{tabular}{ccccccc}
\hline Particle-nucleus & $\mu^{ \pm}-\mathrm{d}$ & $\pi^{ \pm}-\mathrm{d}$ & $\mathrm{K}^{ \pm}-\mathrm{d}$ & $\mathrm{p}-\mathrm{d}$ & $\mathrm{p}-{ }_{\Lambda}^{3} \mathrm{H}$ & $\mathrm{p}-{ }_{\Lambda}^{5} \mathrm{He}$ \\
\hline$\Delta$ & \pm 0.01160 & \pm 0.01507 & \pm 0.04535 & 0.07258 & 0.16800 & 0.05276 \\
$\Delta_{z r}$ & \pm 0.00799 & \pm 0.01038 & \pm 0.03123 & 0.04998 & 0.14414 & 0.03610 \\
$\frac{m_{23}}{m_{3}} \kappa, \mathrm{fm}^{-1}$ & 0.46289 & 0.46289 & 0.46289 & 0.46289 & 0.18325 & 1.61031 \\
$1 / a_{B}, \mathrm{fm}^{-1}$ & 0.00370 & 0.00480 & 0.01446 & 0.02314 & 0.02641 & 0.05813 \\
$p_{0}^{b}, \mathrm{fm}^{-1}$ & 0.06870 & 0.07830 & 0.13582 & 0.17182 & 0.11131 & 0.34356 \\
\hline
\end{tabular}




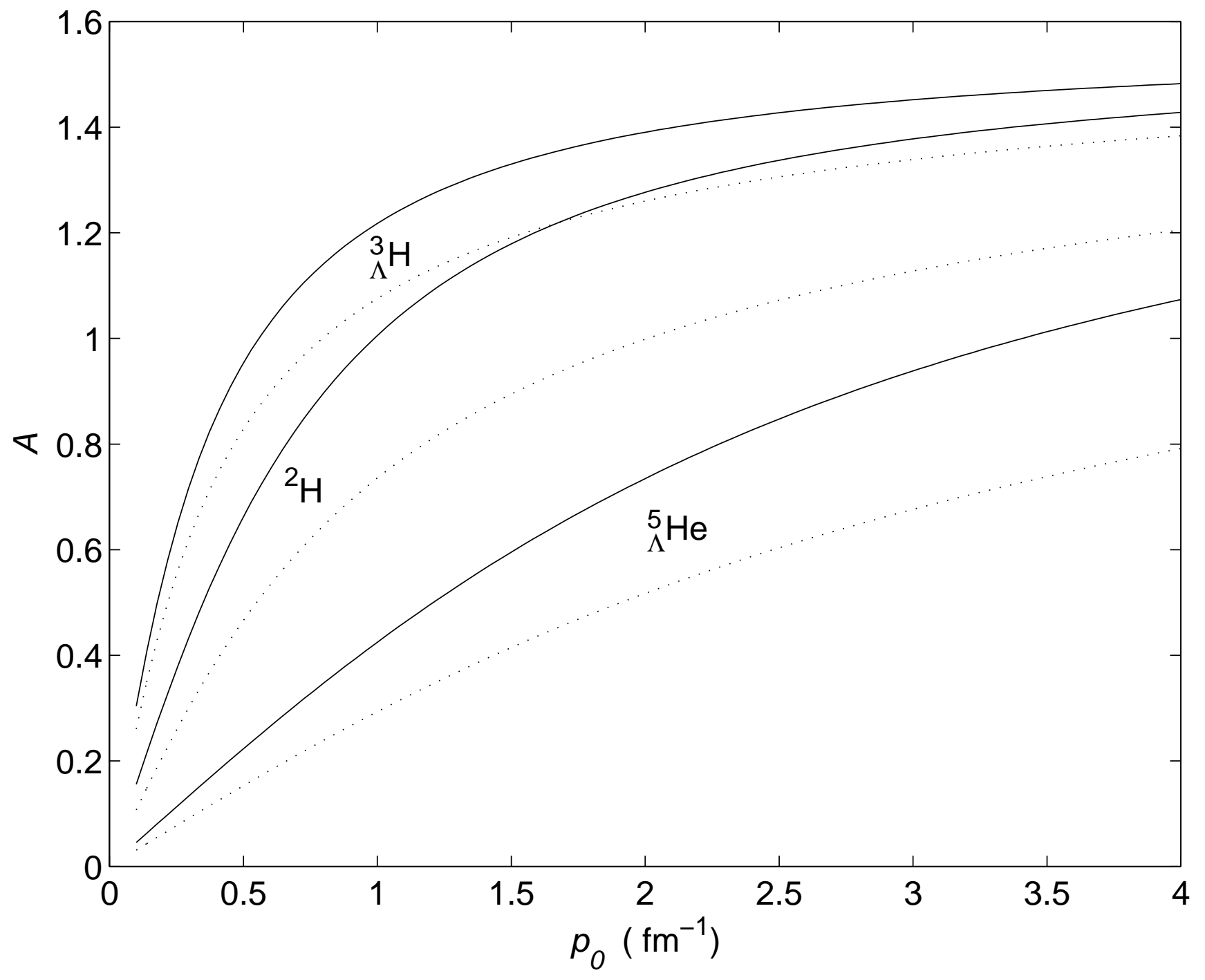




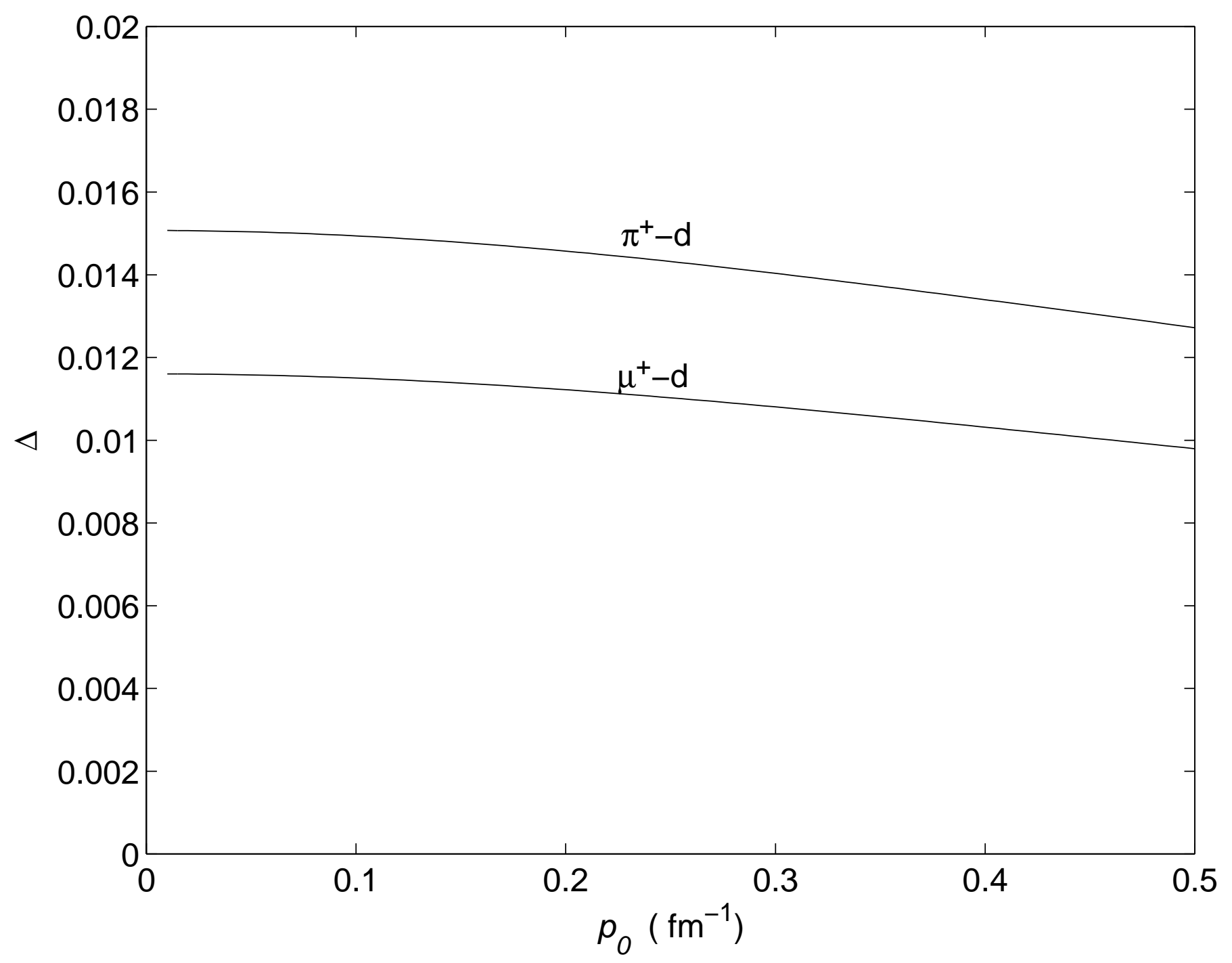




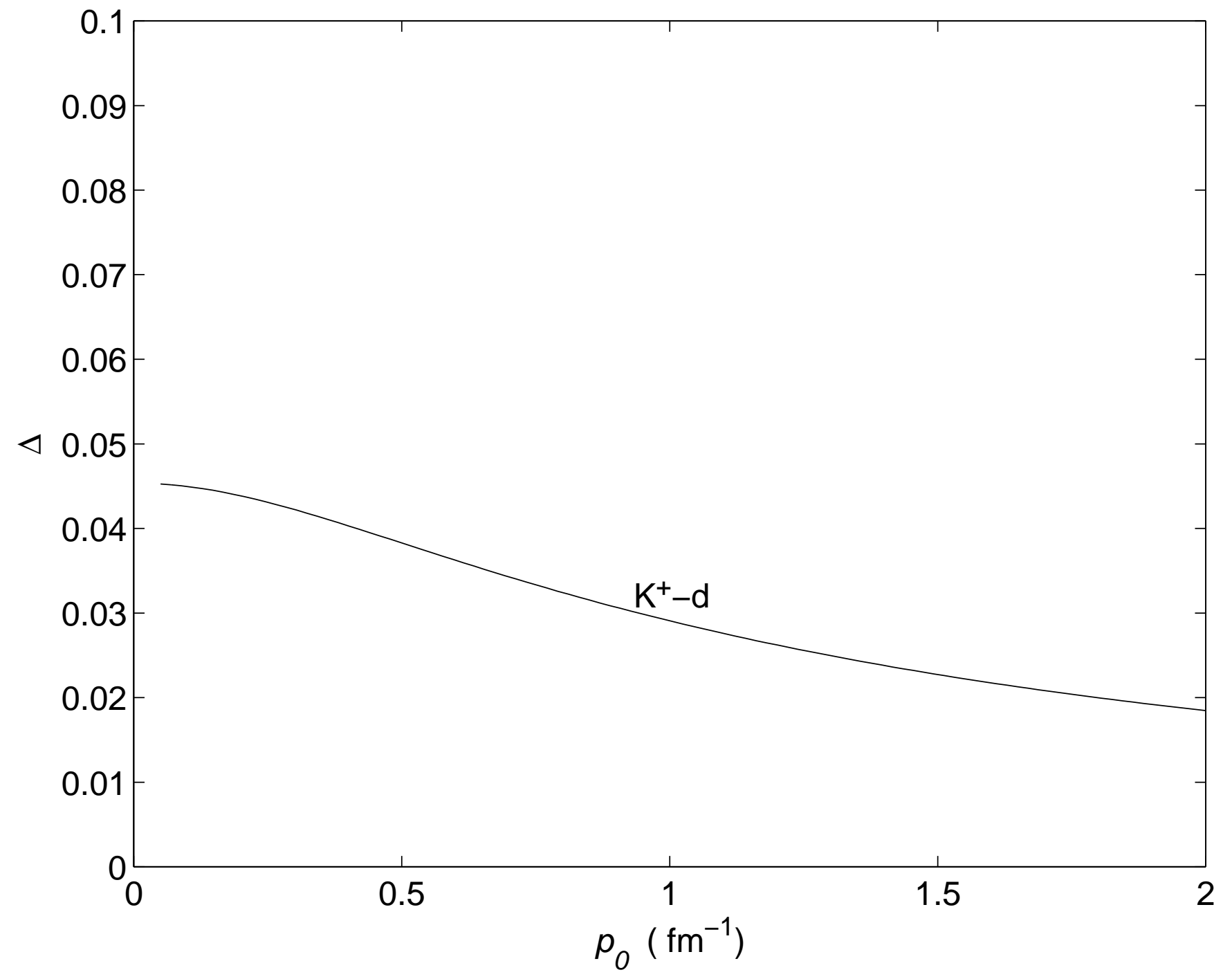




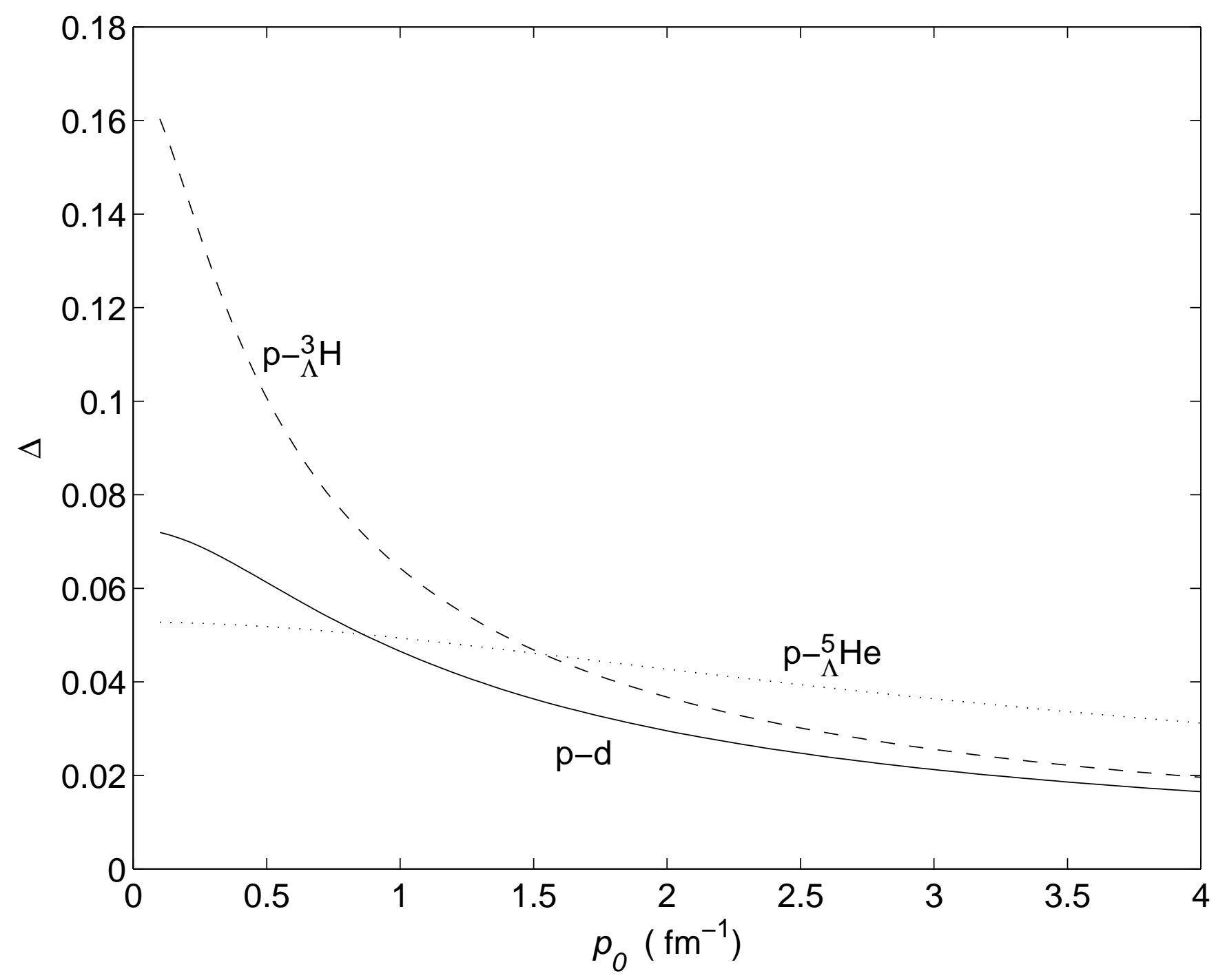

